\title{
SCA7 mice recapitulate CNS, PNS and retina pathologies and show a transcriptional signature of Purkinje cell dysfunction prevailing in SCA1 and SCA2 mice.
}

\section{Anna NIEWIADOMSKA-CIMICKA}

Institut de Genetique et de Biologie Moleculaire et Cellulaire

\section{Frédéric Doussau}

CNRS UPR3212, Universite de Strasbourg

\section{Jean-Baptiste Perot}

Commissariat a l'energie atomique et aux energies alternatives Molecular Imaging Research Center

\section{Michel J Roux}

Institut de Genetique et de Biologie Moleculaire et Cellulaire

\section{Céline Keime}

Institut de Genetique et de Biologie Moleculaire et Cellulaire

\section{Antoine Hache}

Institut de Genetique et de Biologie Moleculaire et Cellulaire

\section{Françoise Piguet}

Institut de Genetique et de Biologie Moleculaire et Cellulaire

\section{Ariana Novati}

Universitatsklinikum Tubingen Institut fur Medizinische Genetik und angewandte Genomik

\section{Chantal Weber}

Institut de Genetique et de Biologie Moleculaire et Cellulaire

\section{Binnaz Yalcin}

Institut de Genetique et de Biologie Moleculaire et Cellulaire

\section{Hamid Meziane}

Institut Clinique de la Souris

\section{Marie-France Champy}

Institut clinique de la souris

\section{Erwan Grandgirard}

Institut de Genetique et de Biologie Moleculaire et Cellulaire

\section{Alice Karam}

Institut de Genetique et de Biologie Moleculaire et Cellulaire

\section{Nadia Messaddeq}

Institut de Genetique et de Biologie Moleculaire et Cellulaire 


\section{Aurélie Eisenmann}

Institut de Genetique et de Biologie Moleculaire et Cellulaire

\section{Emmanuel Brouillet}

Commissariat a l'energie atomique et aux energies alternatives Molecular Imaging Research Center

\section{Hoa Huu Phuc Nguyen}

Institute of mecical Genetics and Applied Genomics, University of Tuebingen

\section{Julien Flament}

Commissariat a l'energie atomique et aux energies alternatives Molecular Imaging Research Center

\section{Philippe Isope}

CNRS UPR3212, Université de Strasbourg

\section{Yvon Trottier ( $\nabla$ Yvon.Trottier@igbmc.fr)}

Institut de Genetique et de Biologie Moleculaire et Cellulaire https://orcid.org/0000-0001-7230-4810

\section{Research article}

Keywords: Spinocerebellar ataxia type 7, Gene deregulation, Purkinje cells, Polyglutamine expansion, Cerebellum, Peripheral neuropathy, Retinopathy

Posted Date: May 13th, 2020

DOl: https://doi.org/10.21203/rs.3.rs-27474/v1

License: (c) (i) This work is licensed under a Creative Commons Attribution 4.0 International License. Read Full License

Version of Record: A version of this preprint was published at The Journal of Neuroscience on April 22nd, 2021. See the published version at https://doi.org/10.1523/JNEUROSCI.1882-20.2021. 


\section{Abstract}

Background Background: Spinocerebellar ataxia type 7 (SCA7) is primarily characterized by progressive cerebellar degeneration with major alteration of Purkinje cells (PC) due to polyglutamine expansion in ATXN7, a subunit of SAGA transcriptional co-regulator complex. Additional neural tissues are affected and contribute to diverse symptoms. Current animal models were insufficient to recapitulate the broad spectrum of SCA7 pathology and the mechanisms of PC degeneration remain poorly explored. Methods: To delineate spatio-temporal features of SCA7, we performed a longitudinal characterization of a new knock-in mice line expressing ATXN7 with 140 glutamines (SCA7 140Q/5Q ) using a battery of analyses including motor tests, brain and retina imaging, morphometry, electrophysiology, electron microscopy and immunohistochemistry of disease tissues. We also determined the cerebellar transcriptome and cell-type specific gene deregulation. Results: Here we describe the first SCA7 knock-in mice that combine most cardinal features of SCA7, including retinal, cerebellar, cerebral and peripheral nerves pathologies, which account for progressive impairment of behavior, motor and vision functions. MRI and brain morphometry reveal atrophy of grey and white matter of specific cerebral regions, while peripheral nerves and photoreceptors show functional and morphological alterations. We show that cerebellar pathology is characterized by gene deregulation in all cerebellar cell types and alterations of SAGA-dependent epigenetic marks. Intranuclear accumulation of mutant ATXN7 and gene downregulation precede the onset of PC pacemaker dysfunction. Interestingly, PC show loss of expression of 83 PC-specific genes coding for ion channels, receptors and signaling proteins involved in pacemaker function and long-term depression, which are causal factors of many type of ataxias. Comparison of cerebellar transcriptome with other SCAs reveals a subset of 67 PC-specific genes downregulated in SCA1, SCA2 and SCA7, providing a common signature of early PC dysfunction. Conclusions: The SCA7 140Q/5Q mice represent a promising model for the investigation of different aspects of SCA7 pathogenesis, and offers opportunities for translational development of therapeutic strategies by targeting the brain, retina, peripheral nerve or whole body. Our results also provide insight into converging mechanisms of PC degeneration in polyglutamine SCAs and point out molecular targets for therapeutic development $w$ hich may prove beneficial for several SCAs.

\section{Background}

Spinocerebellar ataxia type 7 (SCA7) belongs to the large family of autosomal dominant cerebellar ataxias, a heterogeneous group of neurodegenerative diseases primarily characterized by progressive damage of the cerebellum and its associated tracts leading to inability to coordinate balance, gait, and speech. SCA7 is also clinically characterized by spastic ataxia gait, dysphagia, intentional tremors, slow eye movement, ophthalmoplegia, as well as pyramidal signs $(1,2)$. Decreased visual acuity distinguishes SCA7 from other inherited ataxias $(2,3)$.

Post-mortem cerebellum analysis of SCA7 patients shows important loss of Purkinje cells (PC) (3-5). Neuronal loss is also observed in the granule cell layer, the inferior olivary nuclei and the basis pontis associated with the atrophy of spinocerebellar and pyramidal tracts (6). There is atrophy or loss of 
myelinated fibers in the cerebellar white matter and extracerebellar associated structures including the spinal cord (2-5). Post-mortem brains analysis also shows widespread neurodegeneration in additional structures, including the telencephalon, midbrain and medulla oblongata (4). Brain imaging reveals the prominent atrophy of the cerebellum's grey and white matters and the pons, a correlation between cerebellar volume decrease and motor impairment, and more prominent degeneration in lobule IX and X of cerebellum compared to other structures $(1,3,5,7-11)$. Neurophysiological studies suggest the existence of a critical sensorimotor peripheral neuropathy $(5,12,13)$. Visual impairment is mainly due to severe loss of cone and rod photoreceptors $(5,14)$.

SCA7 is part of a group comprising SCA1, SCA2, SCA3, SCA6 and SCA17 as well as dentatorubropallidoluysian atrophy, spinal bulbar muscular atrophy and Huntington's disease that are caused by the expansion of a CAG repeat sequence located within the coding region of specific genes. SCA7 is caused by CAG repeat expansion in the $A T X N 7$ gene that is translated into a polyglutamine (polyQ) tract in ataxin-7 (ATXN7) protein (15). SCA7 expansions range from 37 to more than 200 repeats (16). The length of the expanded repeats correlates inversely with the onset of symptoms and positively with disease severity and progression. Very large expansions ranging from 200-460 repeats cause infantile-onset SCA7, which affects neural and non-neural tissues and leads to early death (17-21). ATXN7 is a ubiquitously expressed nucleo-cytoplasmic protein regulated by nuclear import and export signals and alternative splicing, suggesting its involvement in multiple functions (16). Related to the nucleus, ATXN7 is part of Spt-Ada-Gcn5 Acetyltransferase (SAGA) (22), a multiprotein complex that regulates transcription through histone modifying activities: the acetylation of lysine 9 of histone H3 (H3K9ac) and the deubiquitination of lysine 120 of $\mathrm{H} 2 \mathrm{~B}$ (H2Bub). Studies in zebrafish indicate that Atxn7 is involved in the differentiation of photoreceptor and PC, two highly vulnerable neurons in $\operatorname{SCA7}(23,24)$.

Several mouse models have been generated over the past years to study the pathogenesis of SCA7 (25). While transgenic mice overexpressing one isoform of mutant ATXN7 (mATXN7) under heterologous promoters helped to generate robust phenotypes and identify disease mechanisms (16), they presented limited features of the disease and may have masked early cellular and molecular alterations important to pathogenesis due to overexpression. In contrast, knock-in mice harboring 266 CAG repeat expansion in the mouse Atxn 7 locus (named SCA $7^{266 \mathrm{Q} / 5 \mathrm{Q}}$ mice) have provided a genetically and physiologically more representative disease model, and proved useful to uncover alteration of short term plasticity of hippocampal neurons (26). However, except for the retinopathy, the functional, neuropathological and molecular alterations underlying motor incoordination and degeneration of PC of SCA7 ${ }^{266 Q / 5 Q}$ mice remain poorly characterized. One explanation might be that $S C A 7^{266 Q / 5 Q}$ mice are extremely difficult to expand and manage for in-depth characterization due to the severe phenotype, early death (14-19 weeks), poor male fertility and very small litters. A cognate SCA $7^{100 Q / 50}$ line, which was obtained by intergenerational contraction of 266 CAG repeat expansion, have half-life of $\sim 19$ months and did not go through deep characterization (27).

To assess emerging preclinical therapeutic strategies, disease models must recapitulate all major clinical human features, and present correlative phenotypic, functional, neuropathological and molecular 
alterations with defined onset and progression. We describe here a new SCA7 knock-in mice line carrying 140 CAG repeats, SCA $7^{140 Q / 5 Q}$ (which is derived from the SCA7 ${ }^{266 Q / 5 Q}$ mouse model by CAG repeat contraction), that combines the major clinical features observed in patients, including retinal, cerebellar, cerebral and peripheral nerves pathologies. A comprehensive characterization of this model, performed using a battery of analyses (motor tests, retina and brain imaging, electrophysiology of PC, photoreceptors and peripheral nerves, electron microscopy and immunohistochemistry), provides quantitative phenotypic parameters and defined disease onset and progression suitable for pathogenesis study and preclinical trials. Transcriptome profiling allowed to identify cell-type specific gene deregulation in the cerebellum and to highlight PC as the most affected neurons. Strikingly, we found that 67 PCenriched genes critical for spontaneous PC firing and long-term depression (LTD) are downregulated in SCA7 mice as well as in SCA1 and SCA2. Our results provide a common signature of early PC dysfunction and molecular targets for therapeutic development for multiple SCAs.

\section{Methods}

\section{Mouse information.}

The SCA $7^{140 Q / 5 Q}$ knock-in mouse line harbors an expansion ranging from 140-150 CAG repeats in exon1 of the Atxn7 locus. Mice are kept on C57BI/6J background and bred in the Mouse Clinical Institute (Illkirch, France) with a $12 \mathrm{~h}$ light/dark cycle and free access to food and water. Genotyping was performed by PCR as described previously (26). Wild type (WT) and SCA $7^{140 Q / 5 Q}$ males and females were analyzed, unless indicated.

\section{Behavioral and motor performance analysis.}

To ensure the replicability of SCA7 mouse phenotype, two mouse colonies were raised in the husbandry of different research centers and were analyzed using standardized protocols by independent experimenters and instruments. The experiments were all performed during the light phase of a $12 \mathrm{~h}$ light/dark cycle.

Open field test. The open fields were placed in a room homogeneously illuminated at 150 lux. Mice of the first colony were tested in automated open fields ( $44.3 \times 44.3 \times 16.8 \mathrm{~cm}$, Panlab, Barcelona, Spain), virtually divided into central and peripheral regions. Each mouse was placed in the periphery of the open field and allowed to explore freely for $30 \mathrm{~min}$, with the experimenter out of the animal's sight. The distance traveled, the number of rears, average speeds and resting time were automatically recorded and reflect the general locomotor or motor activity phenotype. The number of entries and the time spent in the central and peripheral regions were used as index of emotionality/anxiety. Mice of the second colony were tested in an open field $(46 \times 46 \mathrm{~cm})$. Each mouse was tracked over 30 minutes with EthoVision XT 11 and 12 (Noldus Information Technology, The Netherlands) and automatically analyzed for the same parameters, except for the number of rears that were manually scored. 
Rotarod. To assess the ability to maintain balance, mice of the first colony were tested on a rotating rod of $5 \mathrm{~cm}$ in diameter covered by grey rubber foam (Bioseb, Chaville, France). Mice were given three testing trials during which the rotation speed accelerated from 4 to $40 \mathrm{rpm}$ in $5 \mathrm{~min}$. Trials were separated by a minimum of 5-10 min interval. The average latency before falling from the rod was used as index of motor coordination performance. Mice of the second colony were assessed using an apparatus from TSE Systems (Bad Homburg, Germany) with $3.5 \mathrm{~cm}$ rod diameter without rubber foam, using the same parameters as for the first colony. The floor of the rotarod was covered with soft material to avoid animal discomfort when falling. Light intensity was set to about 115 lux.

Notched bar. The test was used to test hindlimb coordination. On the first day, mice were trained on a 2 $\mathrm{cm}$-wide and $110 \mathrm{~cm}$ long natural wooden notched bar made of 26 platforms of $2 \mathrm{~cm}$ long spaced by 27 gaps of $2 \mathrm{~cm}$ and connected to the goal box $(12 \times 12 \times 14 \mathrm{~cm})$, under 115 lux. The second day mice were tested in the same conditions and time to cross the notched bar was measured. A back paw going through a gap was considered as an error.

Beam walking test. The test consisted of a $110 \mathrm{~cm}$ long wooden beam $(2 \mathrm{~cm}$ diameter), positioned $50 \mathrm{~cm}$ above the floor. One extremity of the beam was used as starting position while the opposite extremity was connected to a goal box (of $12 \times 12 \times 14 \mathrm{~cm}$ for the first colony, and $8.5 \times 8.5 \times 12 \mathrm{~cm}$ for the second colony). The floor of the goal boxes was covered with clean sawdust. The floor under the beam was covered with soft material. During the whole protocol, the light intensity in proximity of the beam was maintained at about 115 Lux. Each mouse was first habituated to the goal box for 1 minute before three training and three testing trials. On the first training trial, mice were placed on the beam near and facing the goal box and allowed to access it. On the second and third training trials, mice were placed further away (33- and 66-cm, respectively) facing the box and were allowed to reach and enter the box. On the testing sections, mice were placed at the beam extremity $(100 \mathrm{~cm}$, starting position), facing the box and were allowed to reach the goal box. After each training and testing trial, mice were left for 15 seconds in the goal box. The latency to reach the box (cut off 60 seconds) and the number of errors (slips) while crossing the beam were recorded.

Footprint. After coating the front- and hind feet with 2 different colors of non-toxic inks, mice walked through a tunnel (length: $50 \mathrm{~cm}$, width: $9 \mathrm{~cm}$, height: $6 \mathrm{~cm}$ ) covered with paper. The following five footprint patterns were manually measured: matching between paws, stride length, stance width, distance between fore- and hindlimbs.

Grip strength test. This test measures the maximal muscle strength (g) using an isometric dynamometer connected to a grid (Bioseb). Mice were held by the tail and allowed to grip the metal grid with the 4 paws, then they were pulled backwards until they released it. Each mouse was submitted to 3 consecutive trials and the maximal strength developed by the mouse before releasing the grid was recorded. The average value of the three trials adjusted to body weight was used as index of the muscle strength.

CatWalk. Gait analyses were performed using CatWalk XT 8.1 (Noldus Information Technology, Wageningen, The Netherlands). The CatWalk apparatus consists of a closed corridor resting on a glass 
plate. The walkway $(20 \mathrm{~cm})$ is illuminated with red light from the ceiling and green light from the edges of the plate (green intensity threshold: 0.30 ; camera gain: 20 ). When an animal enters the corridor, the light reflected by the paws is detected by a camera placed $25 \mathrm{~cm}$ below the walkway. Each mouse was placed on the walkway at one extremity and allowed to move in the corridor in both directions. Only runs lasting a maximum of 10 seconds with $60 \%$ maximal speed variation were counted. Five successful runs per animal were recorded. The swing (s) defined as the duration of no contact of a paw with the glass plate was analyzed separately for fore- and hindlimbs and averaged between left and right sides.

\section{Optical Coherence Tomography and electroretinography.}

Optical Coherence Tomography (OCT). Pupils were dilated with a bilateral topical application of atropine $(1 \%$, Virbac, France). Animals were then anesthetized using isoflurane (4\% for induction, $2 \%$ for maintenance in a 2 I.min- 1 flow of $50-50 \%$ mix of air and $\mathrm{O}_{2}$ ) and placed on the stage of a Bioptigen R2200 spectral domain OCT (Bioptigen, North Carolina, USA). Cornea was kept moisturized with a thin layer of ocular gel (Ocry-gel, TVM, France) applied with surgical eye spears. For each eye, a rectangular scan was obtained, corresponding to a region of $1.4 \times 1.4 \mathrm{~mm}$ centered on the optic nerve. The thicknesses of the whole retina (including the pigmentary epithelium), the segments, the outer nuclear layer, the inner nuclear layer and the inner plexiform layer for a given eye were calculated as the average of 2 measures performed manually at $0.5 \mathrm{~mm}$ from the optic nerve center using the Bioptigen InVivoVue 2.4.33 software.

Electroretinography (ERG). Pupils were dilated and cornea moisturized as described above. Mice were then anesthetized with an intraperitoneal injection of a mixture of ketamine $(100 \mathrm{mg} / \mathrm{kg})$ and medetomidine (1.2 mg/kg, Domitor ${ }^{\circledR}$, Vétoquinol, France). Mice were placed on a platform on top of a chemical heating pad (Aquapack, China). A DTL thread was used as the active electrode and placed across the cornea. To minimize invasiveness, we replaced the subcutaneous needles traditionally used as reference and ground electrodes, respectively by a gold loop placed in the mouth (around the incisors), and a finger clip electrode placed on the tail with conductive paste. The platform was then moved forward to position the mouse head inside a Ganzfeld with flashes controlled with a Visiosystem (SIEM Bio-médicale, Nice, France). The background light was set at $25 \mathrm{Cd} . \mathrm{m}^{-2}$. Single flash photopic responses were recorded for intensities of 1,3 and $10 \mathrm{Cd} . \mathrm{s.m}^{-2}$, and flicker responses were recorded at frequencies of $2,5,10,20$ and $25 \mathrm{~Hz}$ with $3 \mathrm{Cd} . \mathrm{s}^{-\mathrm{m}^{-2}}$ flashes. The b-wave amplitude was measured offline with the Visiosystem software. At the end of the test, mice received a subcutaneous injection of atipamezole (10 $\mathrm{mg} / \mathrm{kg}$, Antisedan ${ }^{\circledR}$, Vétoquinol, France) to counteract the action of medetomidine, and were placed on a heating plate set at $37^{\circ} \mathrm{C}$ until awakening.

\section{Electrophysiology.}

Electromyography. Recordings were performed using the Natus UltraProS100 apparatus (Mag2Health, France). Mice were anesthetized using intraperitoneal injection with ketamine/xylazine $(130 / 13 \mathrm{mg} / \mathrm{kg})$. Animals were maintained at $37^{\circ} \mathrm{C}$ during the whole experiment until wake up. Latency and amplitude of 
$\mathrm{M}$ and $\mathrm{H}$ waves were recorded in the plantar hind paw muscle after sciatic nerve stimulation (0.1 ms and $8 \mathrm{~mA}$ intensity).

Electrophysiology of PCs. Following anesthesia by isoflurane inhalation, cerebellum extraction was done in ice-cold $\left(\sim 1^{\circ} \mathrm{C}\right)$ artificial cerebrospinal fluid (ACSF) bubbled with carbogen $\left(95 \% \mathrm{O}_{2}, 5 \% \mathrm{CO}_{2}\right)$ containing (in mM): $120 \mathrm{NaCl}, 3 \mathrm{KCl}, 26 \mathrm{NaHCO}, 1.25 \mathrm{NaH}_{2} \mathrm{PO}_{4}, 2.5 \mathrm{CaCl}_{2}, 2 \mathrm{MgCl}_{2}, 10$ D-glucose and $0.05 \mathrm{mM}$ minocyclin. Cerebella were sectioned (Microm HM650V, Germany) in an ice-cold low-sodium and zerocalcium slicing buffer containing (in mM): 93 N-Methyl-D-Glucamine, $2.5 \mathrm{KCl}, 0.5 \mathrm{CaCl}, 10 \mathrm{MgSO}, 1.2$ $\mathrm{NaH} 2 \mathrm{PO} 4,30 \mathrm{NaHCO}$, 20 HEPES, 3 Na-Pyruvate, 2 Thiourea, 5 Na-ascorbate, 25 D-glucose and 1 Kynurenic acid. Sagittal slices $300 \mu \mathrm{m}$ thick were immediately transferred in bubbled ACSF for 30 minutes at $34^{\circ} \mathrm{C}$ and maintained at room temperature $\left(\sim 25^{\circ} \mathrm{C}\right)$ in bubbled ACSF for at least 1 hour before transfer in a recording chamber, continuously perfused with bubbled ACSF. In order to block inhibitory and excitatory synaptic transmission, blockers of $\mathrm{GABA}_{\mathrm{A}^{-}}$-receptors (100 $\mu \mathrm{M}$ picrotoxin) and ionotropic glutamate receptors ( $1 \mathrm{mM}$ kynurenic acid) were added in ACSF. PCs located in lobules VI or IX/X were visually identified under infrared illumination. All recordings were performed at near physiological temperature $\left(32 \pm 0.5^{\circ} \mathrm{C}\right)$ using an AxoPatch 200A (Molecular Devices, USA). PC discharges were monitored using extracellular recordings with pipettes filled with $0.5 \mathrm{M} \mathrm{NaCl}$ (resistance: 15-30 MW). Data were acquired using WinWCP 4.5.4 freeware (John Dempster, University of Strathclyde, Glasgow, UK; RRID:SCR_014713). Recordings were sampled at $50 \mathrm{kHz}$ and filtered at $2 \mathrm{kHz}$. In extracellular recordings, action potentials were detected using OpenElectrophy, an open source software (http://neuralensemble.org/OpenElectrophy/; RRID:SCR_000819) (Garcia and Fourcaud-Trocme', 2009). Data were stored in SQL databases (Oracle). Spike trains were analyzed using Python software written inhouse (SynaptiQs developed by Antoine Valera) and custom-based routines. PC discharge irregularity was assessed by two parameters: (1) the mean of interspike intervals (ISIs); (2) coefficient of variation for adjacent ISIs (CV2) calculated as follows:

$$
C V_{2}=2 \frac{\left|I S I_{n+1}-I S I_{n}\right|}{\left|I S I_{n+1}+I S I_{n}\right|}
$$

where $I S I_{n}$ and $I S I_{n+1}$ are two consecutive ISI. An average of CV2 over all consecutive pairs of ISI's estimates the intrinsic variability of PC pacemaking activity. This parameter is independent of slow variations in the firing pattern.

\section{Neuroanatomical study.}

Neuroanatomical study was carried out using 10 mice at 33 weeks ( $n=5$ per group) and 12 mice at 50 weeks ( $n=6$ per group). Brain samples were fixed in $4 \%$ buffered formalin for 48 hours. Seventy-eight brain parameters, made of area and length measurements, were taken blind to the genotype across three coronal sections as described (28). Data were analyzed using a t-test with equal variances to determine whether a brain region is associated with neuroanatomical defect or not. 


\section{Magnetic resonance imaging (MRI).}

After paraformaldehyde (PFA) perfusion of 25-week-old mice, brains were removed and stained for one week in a solution of Gadolinium (Dotarem $\odot$, Guerbet, France) in PBS at $2.5 \mathrm{mM}$. This protocol enhances the signal- and contrast-to-noise ratios on MR images of fixed brains (29), Ex vivo MRI experiments were performed on a horizontal 11.7 T Bruker scanner (Bruker, Ettlingen, Germany). A quadrature cryoprobe (Bruker, Ettlingen, Germany) was used for radiofrequency transmission and reception. High-resolution

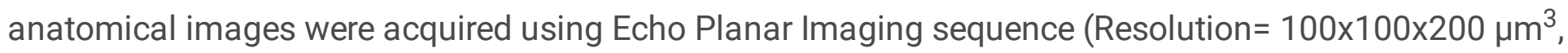
$\mathrm{TR}=3000 \mathrm{~ms}, \mathrm{TE}=28 \mathrm{~ms}$ ). In order to preserve integrity to avoid deformations, brains were kept inside skulls for ex vivo MRI experiments. Anatomical images were co-registered in the same space to create a study template. Transformations to match the study template to an atlas composed of a high-resolution template and a map of region's labels adapted from the Allen mouse brain Atlas (30) were calculated. Finally, transformations to match the study template and labels to each individual anatomic image were calculated. The automated segmentation pipeline was performed using an in-house python library (Sammba-MRI, https://github.com/sammba-mri/sammba-mri).

\section{Immunofluorescence.}

Cryosections. Mice were transcardialy perfused with 1xPBS followed by $4 \%$ PFA/1xPBS. Brains and eyecups were immediately dissected and incubated in $4 \%$ PFA/1xPBS overnight $(0 / n)$ or for $2 h$, respectively. They were washed 3 times in 1 xPBS before a $24 \mathrm{~h}$ incubation in $30 \%$ sucrose for cryoprotection and then mounted in Shandon Cryomatrix embedding resin (ThermoFisher) and immediately frozen. 14-20 $\mu \mathrm{m}$ brain sagittal and $12 \mu \mathrm{m}$ eyecup cryosections (Leica CM 3050S) were collected and stored at $-80^{\circ} \mathrm{C}$.

Vibratome sections. Brains were fixed as described above, washed 3 times in 1xPBS and mounted in $2 \%$ agarose/1xPBS. Vibratome sagittal sections (Leica VT 1000S) of $50 \mu \mathrm{m}$ were collected, washed in $1 \mathrm{xPBS}$, next in $20 \%$ glycerol/1xPBS and stored in $60 \%$ glycerol $/ 1 \mathrm{xPBS}$ at $-20^{\circ} \mathrm{C}$. The $60 \%$ glycerol $/ 1 \mathrm{xPBS}$ was washed out with 1 wash of $20 \%$ glycerol/PBS and 3 washes of 1 xPBS before immunofluorescence.

Cryosections were permeabilized in PBSTx (1xPBS/0.1\% Triton X-100) and blocked for $1 \mathrm{~h}$ at room temperature (RT) with PBSTx supplemented with $7 \%$ deactivated fetal calf serum (FCS). Sections were incubated $\mathrm{o} / \mathrm{n}$ at $4^{\circ} \mathrm{C}$ with primary antibodies in a blocking buffer using the following dilutions: 1:20 000 (cryosections) or 1:5 000 (floating sections) anti-CALB1 (rabbit, D28k, Swant, CB38a); 1:5 000 (cryosection) or 1:1 000 (floating section) anti-CALB1 (mouse, D28k, Sigma, C9848); 1:1 000 (floating sections) vGLUT2 (Guinea Pig, Merck, AB2251-I); 1:1 000 anti-ATXN7 (rabbit, 1261; IGBMC); 1:700 antiGFAP (mouse, Sigma, G3893). Secondary goat anti-rabbit, goat anti-mouse, goat anti-guinea pig IgG antibodies (ThermoFisher, dilution 1:1000) conjugated with Alexa 555, Alexa 488 and Alexa 488 fluorophores, respectively, were applied for $1 \mathrm{~h}$ at RT and subsequently washed 3 times in PBSTx. Nuclei were counterstained with $1 \mu \mathrm{g} / \mathrm{ml}$ DAPI (4',6-diamidino-2-phenylindole dihydrochloride). Controls without 
primary antibodies were performed in parallel. Slides were mounted with Aqua Poly Mount (Polysciences), dried and stored at $+4^{\circ} \mathrm{C}$ protected from light.

Floating cerebellar sections were processed as described above with the following modifications. Sections were incubated in Eppendorf tubes for 3 days at $4^{\circ} \mathrm{C}$ with primary antibodies in $300 \mu \mathrm{l}$ of blocking buffer. Secondary antibodies were applied for $2 \mathrm{~h}$ at RT. Sections were positioned on slides directly before mounting.

Retina images were acquired on a Leica DM 4000B with a Cool SNAP HQ2 camera with 40x objective with zoom factor 2.5 and processed with Fiji. Cerebellum images were acquired either on a confocal microscope (SP8, Leica) with 40x/1.3 oil objective with a zoom factor 1 or on a Spinning disc with Leica DMI 8 inverted microscope equipped with Hamamatsu Orca flash 4.0 Camera 5 (Gataca Sytem) connected with Metamorph software (Molecular Devices). For the acquisitions, HC PL APO 40x/1.3 oil objective and three diode lasers $(405,488$, and $561 \mathrm{~nm})$ were used. Image brightness and contrast were equivalently adjusted in Fiji for display purposes when necessary.

\section{PC size and circularity measurements.}

Sagittal floating sections $(50 \mu \mathrm{m})$ of central vermis were immunostained with anti-Calbindin 1 antibody and acquired using confocal SP8 (Leica) microscope. The obtained z-stacks were subdivided into 3 groups of 15 slices using Fiji. After background subtraction the PC layer was outlined manually in the vicinity of PC somas. Next, a customized Fiji macro allowing an automatic detection and measurement the PC area and circularity was applied. The macro was kindly provided by Stephane Collins (Inserm UMR1231 GAD, Dijon, France). Manuel correction was performed dendritic parts were identified as PC. Edge PC were excluded from measurements.

\section{Morphometry of cerebellar vermis sections.}

Sagittal $20 \mu \mathrm{m}$ sections of central vermis were immunostained with anti-CALB1 antibody and counterstained with DAPI. Sections were scanned with Digital Slide Scanner Nanozoomer 2.0 HT (Hamamatsu) using 40x magnification. Images of the cerebellum were visualized using NDPI viewer and exported with $5 x$ magnification into tiff format. Length and width of cerebellar sections as well as the thickness of the molecular cell layer were measured using Fiji software.

\section{vGLUT2 density, volume and extension in the molecular cell layer.}

Sagittal floating sections $(50 \mu \mathrm{m})$ of central vermis were immunostained with anti-Calbindin 1 and antivGLUT2 antibodies, counterstained with DAPI. Sections were acquired using YoKogawa CSU W1 Spinning disc with Leica DMI 8 inverted microscope and Hamamatsu Orca flash 4.0 Camera (Gataca Sytem) and a driver by the Metamorph software (Molecular Devices). For the acquisitions, HC PL APO 40x/1.3 oil objective and three diode lasers $(405,488$, and $561 \mathrm{~nm})$ were used. Stacks consisted of $225 \mathrm{z}$-slices with $0.2 \mu \mathrm{m}$ z-step using $6.5 \mu \mathrm{m} \times 6.5 \mu \mathrm{m}$ pixel size. 
Images were saved in tiff format. Subsequently, RGB channels were split in Fiji. Channel with vGLUT2 acquisition («.ims» format) was exported to Imaris v 9.3.1 (Bitplane AG) for 3D reconstruction. The region of interest, namely the molecular cell layer, was selected and 3D volumes were created with the surface module of Imaris. Next, vGLUT2 was analyzed only on this region with the Imaris spot module. The detection of objects in 3-dimensional space was based on both intensity and size (with the estimated XY diameter of $1 \mu \mathrm{m}$ ). Only spots ranging from 0.52 to $30 \mu \mathrm{m}^{3}$ were selected to eliminate background. Density and volume measurements of spots were analyzed in Excel and GraphPad Prism 6. The extension of vGLUT2-positive climbing fiber terminals along the dendrites of PC was measured using Fiji. Namely, the ratio of the extension of the 3D reconstructed vGLUT2 spots in the molecular cell layer over the total length of the molecular cell layer was calculated for the central part of lobule VI and for the side part of lobule X.

\section{Histology and electron microscopy analysis.}

Eyes were prepared for histology and electron microscopy as in (31). Semi-thin (2 $\mu \mathrm{m})$ sagittal sections were cut with an ultramicrotome (Leica Ultracut UCT) and stained with toluidine blue, and histologically analyzed by light microscopy. For sciatic nerve preparation, animals were perfused with $5 \mathrm{~mL}$ of PBS and $10 \mathrm{~mL} 4 \%$ PFA in saline, and tissues were fixed in $2.5 \%$ PFA/2.5\% glutaraldehyde in cacodylate buffer. Ultra-thin $(70 \mathrm{~nm})$ sections were cut and contrasted with uranyl acetate and lead citrate and examined at $70 \mathrm{kv}$ with a Morgagni 268D electron microscope. Images were captured digitally by Mega View III camera (Soft Imaging System) and contrast was adjusted for display purposes.

\section{Western blot analysis.}

Cerebellum and retina were dissected and snap frozen in liquid nitrogen. Their whole cell extracts were obtained by lysis and sonication of the tissue in, respectively, $500 \mu \mathrm{l}$ and $150 \mu \mathrm{l}$ of the buffer containing $50 \mathrm{mM}$ Hepes-KOH (pH 7.5), $140 \mathrm{mM} \mathrm{NaCl}, 1 \mathrm{mM}$ EDTA, $0.1 \%$ Na-deoxycholate, $1 \%$ SDS, $1 \%$ Triton-X100, $1 \mathrm{x}$ Protease Inhibitor Cocktail (Roche), $20 \mathrm{mM}$ N-ethylmaleide, $20 \mathrm{mM}$ nicotinamide. The last two compounds were not used for ATXN7 detection. Protein concentrations were measured using Bradford protein assay. $10 \mu \mathrm{g}$ (histone analysis) and $30 \mu \mathrm{g}$ (ATXN7 analysis) of total proteins were used for electrophoresis. Electrophoresis was run in 4-12\% Bis-Tris Protein Gels in NuPAGE MOPS SDS Running Buffer (ThermoFisher) for histone analysis and in NuPAGE ${ }^{\text {TM }} 3-8 \%$ Tris-Acetate Protein Gels in NuPAGE ${ }^{\text {TM }}$ Tris- $^{-}$ Acetate SDS Running Buffer (ThermoFisher) for ATXN7 detection. Following electrophoresis, proteins were transferred on nitrocellulose membrane for $1.5 \mathrm{~h}$ at $200 \mathrm{~mA}$ or overnight at $30 \mathrm{~mA}$. Antibodies were diluted in $1 \times$ PBSTM (1xPBS with $0.1 \%$ Tween, and $5 \%$ non-fat milk). The following primary antibodies were applied overnight at $4^{\circ} \mathrm{C}$ : anti-ATXN7, 1:1 000 (ThermoFisher, PA1-749); anti-H2B, 1:10 000 (IGBMC clone H2-2A8); anti-H2Bub 1:2 000 (MediMabs, MM-0029); anti-H3K9ac 1:2 000 (Abcam ab4441); antiGAPDH 1:10 000 (Merck Millipore, MAB374); anti-TUBB 1:2 000 (IGBMC, 2A2). Secondary antibodies: Peroxidase AffiniPure $F(a b ') \otimes$ Fragment Goat Anti-Rabbit IgG (H+L) (GARPO; 1:10 000, Jackson ImmunoResearch Lab.) and Peroxidase AffiniPure Goat Anti-Mouse IgG (H+L) (GAMPO; 1:10 000, Jackson ImmunoResearch Lab.) were applied in 1x PBSTM for $2 \mathrm{~h}$ at RT, washed three times in 1xPBST, 
and proteins were detected using SuperSignal West Pico PLUS Chemiluminescent kit (ThermoFisher). Chemiluminescent signal was acquired on Amersham Imager 600. Images were analyzed using Fiji.

\section{RNA-seq library preparation and sequencing.}

RNA-Seq libraries were prepared from $300 \mathrm{ng}$ of total RNA using TruSeq Stranded mRNA Library Prep Kit and TruSeq RNA Single Indexes kits A and B (Illumina, San Diego, CA), according to manufacturer's instructions. Briefly, following purification with poly-T oligo attached magnetic beads, mRNA were fragmented using divalent cations at $94^{\circ} \mathrm{C}$ for 2 minutes. RNA fragments were reverse transcribed into first strand CDNA using random primers. Strand specificity was achieved by replacing dTTP with dUTP for the second strand cDNA synthesis using DNA Polymerase I and RNase $\mathrm{H}$. Following addition of a single ' $A$ ' base and subsequent ligation of the adapter on double stranded $c D N A$ fragments, products were purified and amplified by PCR ( $30 \mathrm{sec}$ at $98^{\circ} \mathrm{C}$; [ $10 \mathrm{sec}$ at $98^{\circ} \mathrm{C}, 30 \mathrm{sec}$ at $60^{\circ} \mathrm{C}, 30 \mathrm{sec}$ at $72^{\circ} \mathrm{C}$ ] $12 \mathrm{cycles}$; 5 min at $72^{\circ} \mathrm{C}$ ) to create the CDNA library. Surplus PCR primers and adapter dimers were removed by purification using AMPure XP beads (Beckman-Coulter, Villepinte, France) and the final cDNA libraries were checked for quality and quantified using capillary electrophoresis. Libraries were sequenced on Illumina Hiseq 4000 sequencer as Single-Read 50 base reads following Illumina's instructions. Image analysis and base calling were performed using RTA 2.7.3 and bcl2fastq 2.17.1.14. Adapter dimer reads were removed using DimerRemover (https://sourceforge.net/projects/dimerremover/)

\section{RNA-seq data analysis.}

Image analysis and base calling were performed using RTA 2.7.3 and bcl2fastq 2.17.1.14. Reads were mapped onto the mm10 assembly of mouse genome using Tophat v2.0.14 (32) with bowtie v2.1.0 aligner. Gene expression was quantified using HTSeq v0.6.1 (33) and gene annotations from Ensembl release 86. Statistical analysis was performed using R and DESeq2 v1.6.3 Bioconductor library (34). Gene functional annotations were performed using Ingenuity Pathway Analysis (IPA) and String v10.5 software $(35,36)$.

\section{Analysis of public datasets.}

For SCA1 dataset (GEO: GSE75778), FASTQ files were retrieved from Sequence Read Archive using fastqdump (SRA Toolkit v2.8.0, BioProject PRJNA305316, samples: GSM1967518-GSM1967520; GSM1967524- GSM1967529; GSM1967533 - GSM1967538; GSM1967542 - GSM1967544), and only the first 50 bases of read 1 sequences were retained for further analysis. For SCA2 dataset (Array Express: EMTAB-6293), FASTQ files were retrieved from Sequence Read Archive using fastq-dump (SRA Toolkit v2.8.0, BioProject PRJEB24319, samples ERR2237861-ERR2237880, ERR2237882 - ERR2237893). Reads were then mapped onto the mm10 assembly of mouse genome using Tophat v2.0.14 (32) with bowtie v2.1.0 aligner. Gene expression was quantified using HTSeq v0.6.1 (33) and gene annotations from Ensembl release 90. Statistical analysis was performed using R and DESeq2 v1.18.1 (34). 
Microarray probe sets of Doyle et al. (37) were converted into gene names according to $\mathrm{mm} 10$ annotation using the Affymetrix NetAffx Annotation File (Mouse430_2 Annotations, Release 36). Next, the cell-type subdivided datasets were curated to indicate the genes present uniquely in one cell-type dataset and in more than 1 cell-type datasets. The obtained gene lists were used to study cell-type distribution of transcriptional changes in SCA7.

\section{Quantitative RT-PCR.}

Total RNA samples were extracted from 1 cerebellum/sample or 2 retinas/animal/sample using TriReagent (MRC) following the manufacturer's instruction. 500ng of purified RNA was then reverse transcribed using QuantiTect ${ }^{\circledR}$ Reverse Transcription kit (Qiagen) according to the manufacturer's protocol. The real-time qPCR (RT-qPCR) reactions were performed on obtained cDNAs (1/20 dilution) using QuantiTect SYBR Green PCR Master Mix (Qiagen), and specific primers (Additional file 1: Table S1) with a Light Cycler 480 (Roche). Gene expressions were normalized based on the expression of Hprt or 36B4 (Rplp0).

\section{Statistical analysis.}

Information on number $(\mathrm{n})$ values and what $\mathrm{n}$ represents (e.g., animals, biological replicates) and definition of center and dispersion and precision measures [e.g., mean, median, standard error of the mean (SEM)] can be found in figure legends. Data were analyzed using GraphPad Prism 7 or 8 or Stat view. Differences between means were assessed with unpaired Student's $t$ test, one-way analysis of variance (ANOVA) or ordinary two-way ANOVA or two-way ANOVA with repeated measurement, followed by post-hoc testing of pairwise comparisons among genotypes, (Bonferroni correction), as indicated. Differences between median were analyzed using Mann-Whitney test. Survival of mice with different genotype and sex was plotted using the Kaplan-Meier method and statistical comparisons were performed using the Log Mantel-Cox test. Further information are indicated in figure legends and in Additional file 2: Table S2. Significance was established at $p<0.05$. Data are expressed as mean \pm SEM unless indicated.

\section{Results}

\section{General phenotypic features of SCA7 mice carrying 140 CAG repeats.}

Previous SCA $7^{266 Q / 5 Q}$ mice are severely affected and die at about 16 weeks of age (26). SCA7 $140 \mathrm{Q} / 5 \mathrm{Q}$ heterozygous mice have a longer median lifespan of 54 weeks for both males and females (Fig. 1a). SCA $7^{140 Q / 140 Q}$ homozygous mice have a much shorter lifespan than heterozygotes. Age-related body weight increase of SCA $7^{140 Q / 5 Q}$ and SCA $7^{140 Q / 140 Q}$ mice significantly differed from their WT littermates (males $F(11,220)=105, p<0.0001$; females $F(11,242)=36,1, p<0.0001)$. SCA7 ${ }^{140 Q / 5 Q}$ females and males gained weight at the same rate as WT mice up to 12 and 14 weeks, respectively, and then plateaued until 24 weeks with an underweight of $18 \%$ for females and $20 \%$ for males compared to WT of the same sex 
and age (Fig. 1b). Subsequently, the weight of SCA7 ${ }^{140 Q / 5 Q}$ decreased, while remaining stable for WT mice. The body weight of SCA $7^{140 Q / 140 Q}$ mice plateaued earlier at 10 weeks for males and 12 weeks for females, and strongly decreased after 20 weeks. Compared to age-matched WT male and female littermates, the body lengths of adult SCA $7^{140 Q / 5 Q}$ and SCA $7^{140 Q / 140 Q}$ males were significantly shorter by $6.6 \%(p<0.01)$ and $23 \%(p<0.0001)$, respectively, and those of adult SCA7 ${ }^{140 Q / 5 Q}$ and SCA7 ${ }^{140 Q / 140 Q}$ females by $6.5 \%(p<0.05)$ and $21 \%(p<0.0001)$, respectively (Fig. 1c). Further characterization was mainly performed on SCA $7^{140 Q / 5 Q}$ mice, as they represent the genetic status of most SCA7 patients.

Examination of general physical appearance and body shape of $S C A 7^{140 Q / 50}$ mice did not reveal gross morphological abnormalities at 28 weeks of age. The proportion of lean, fat and bone tissues was similar to their WT littermates, when normalizing to body weight (Additional file 3; Figure S1). From about 40 weeks of age, SCA $7^{140 Q / 5 Q}$ mice showed already severe motor deficit and were easily distinguishable from control animals due to their smaller body size and hypoactivity, and some with a hunched posture (kyphosis) and tremors. Because of the poor health status and following recommendation of our animal health regulator, $\mathrm{SCA} 7^{140 Q / 5 Q}$ mice were generally sacrificed at this stage.

\section{Degeneration of photoreceptors, pigmented epithelium and Bruch's membrane.}

To determine the onset and progression of $S C A 7^{140 Q / 50}$ mouse retinopathy, analysis was made by correlating non-invasive OCT and ERG, and histological and molecular analysis. The OCT measurement of 8 different morphological parameters indicated that the overall structure of SCA $7^{140 Q / 50}$ retina was normal at 9 weeks (Fig. 2a-c and Additional file 3; Figure S2). However, from 15 weeks, photoreceptor segment layers progressively got thinner with age as compared to WT littermates (Fig. 2b). The same was observed for the outer nuclear layer (ONL) but occurred later (23 weeks) and not as severely (Fig. 2c). There was no clear scalable alteration of thickness of the inner nuclear layer (INL), the inner plexiform layer (IPL) or any other eye structures in SCA7 mice, except for the anterior chamber that showed a significant reduction from 23 weeks onward (Additional file 3; Figure S2).

ERG studies in SCA7 patients showed that cone dysfunction precedes rod dysfunction (3). We evaluated cone function of WT and SCA $7^{140 Q / 5 Q}$ mice by measuring the cone-derived b-wave amplitudes of photopic (single flashes ERG of 1, 3 and 10 Cd.s. ${ }^{-2}$ ) and flicker responses ( 5 frequencies between 2 and $25 \mathrm{~Hz}$ ) at 5, 10, 15 and 23 weeks. At 5 weeks, b-wave amplitudes of both photopic and flicker responses of SCA $7^{140 Q / 5 Q}$ mice were similar to WT littermates. However, at 10 weeks, the b-wave amplitudes of photopic and flicker responses of SCA $7^{140 Q / 5 Q}$ mice significantly decreased relative to WT mice, between $32 \%$ and $36 \%$ for photopic flashes and between $37 \%$ and $45 \%$ depending on flicker frequency (Fig. $2 \mathrm{~d}-\mathrm{e}$ and Additional file 4: Table S3). The loss of b-wave amplitudes of photopic responses and flicker further worsened with age, with reductions between $81 \%$ and $100 \%$ at 23 weeks.

The green and blue opsin (OPN1MW, OPN1SW, respectively) of cones and the rhodopsin (RHO) of rods are light-sensitive pigments that initiate vision and represent major components of photoreceptor outer 
segments. RT-qPCR showed that expression of these genes progressively decreased with age in SCA $7^{140 Q / 5 Q}$ mice (Fig. 2f). Notably, the level of Opn1mw and Opn 1 sw RNAs already showed about $65 \%$ $(p<0.01)$ and $90 \%(p<0.01)$ reduction, respectively, in 10-week-old mutant mice, when cone-derived ERG responses began to decrease. The decrease of cone opsin RNAs was earlier and more drastic than for the Rho RNA, consistent with the primary alteration of cone function in SCA7 patients. Therefore, the loss of cone ERG of SCA $7^{140 Q / 5 Q}$ mice closely correlates with the loss of cone opsins expression, and both occur before a significant shortening of outer segments detected by OCT.

Histology and electron microscopy indicated that photoreceptors had almost completely lost their outer segments at 44 weeks in SCA $7^{140 Q / 5 Q}$ mice and at 20 weeks in SCA $7^{140 Q / 140 Q}$ mice, while the thickness of inner segments showed only a slight reduction in both SCA7 genotypes, compared to WT retina (Fig. $2 \mathrm{~g}$ and Additional file 3: Figure S2i-k). Additional degenerative signs of SCA7 photoreceptors included disorganization of the remnant outer segments, swollen mitochondria and vesicular membrane accumulation in inner segments, several dark stained inner segments and photoreceptor nuclei (Fig. 2hk), and ectopic localization of photoreceptor nuclei in the subretinal space (data not shown). Interestingly, we found striking anomalies of the retinal pigmented epithelium (RPE) and Bruch's membrane of SCA7 ${ }^{140 Q / 5 Q}$ mice never reported previously to our knowledge. Firstly, the thickness of RPE was clearly increased in SCA7 mice as compared to controls (Fig. 2I). Secondly, on electron microscopy, while the normal RPE (Fig. $2 \mathrm{~m}$ ) contains translucent infolding membranes in contact all along the basement membrane, SCA7 RPE showed discontinuous and opaque infolding membranes, with homogenous deposits between the plasma membrane and the basement membrane (Fig. 2n, o). In addition, the Bruch's membrane of SCA7 retina showed enlargement and disorganization, as compared to WT in which the Bruch's membrane was thin and regular (Fig. 2m-o).

\section{Deficits in motor activity and coordination.}

We performed extensive and longitudinal motor performance analysis of SCA $7^{140 Q / 5 Q}$ and WT male littermates using a variety of motor activity paradigms. The spontaneous activity measured with the open field showed that SCA $7^{140 Q / 5 Q}$ mice become significantly hypoactive at 16 weeks, and hypoactivity worsened with age (Fig. 3a-d). Specifically, they travelled shorter distances in the arena, ran slower, rested longer times, and made less rears than WT littermates. However, SCA $7^{140 Q / 5 Q}$ mice did not show anxiety behavior, since they explored the central area similarly to WT mice (Fig. 3e).

When comparing the mean latency to fall from the accelerated rotarod, SCA $7^{140 Q / 5 Q}$ mice fell earlier than WT mice with statistically significant differences from the age of 24 weeks (Fig. 3f). On the notched bar test, SCA $7^{140 Q / 5 Q}$ mice performed similarly to WT littermates up to 17 weeks (Fig. 3g, h). From 20 weeks, performance worsened relative to age-matched WT mice for the time required and the number of mistakes done when crossing the bar. For beam walking test, SCA $7^{140 Q / 5 Q}$ mice took longer time to cross the bar from 20 weeks (Fig. 3i), while the number of mistakes was not significantly different between genotype (data not shown). 
The forward gait patterns were quantitatively assessed for spatial and temporal gait parameters, both for the fore- and hind-limbs, using respectively footprint and Catwalk, an automated gait analysis system. On footprint, SCA7 and WT mice performed similarly in all gait parameters until 17 weeks. From 21 weeks, SCA $7^{140 Q / 5 Q}$ mice made significantly shorter steps (stride length) with forelimbs and hindlimbs of each body side (Fig. 3j). Similarly, the stance width between forelimbs or between hindlimbs was consistently shorter in SCA7 ${ }^{140 Q / 5 Q}$ mice from 21 weeks (Fig. 3k). Temporal gait analysis as recorded by Catwalk showed that the swing duration of right and left forelimbs were significantly affected in SCA7 ${ }^{140 Q / 5 Q}$ mice from 25 weeks, while the swing duration of hindlimbs were not affected (Fig. 3l, m). Finally, the grip strength test showed that the muscle strength of SCA $7^{140 Q / 5 Q}$ mice normalized to animal body weight was similar to WT mice (Fig. 3n).

Replicability of mouse phenotyping across laboratories has important implications in fundamental research and preclinical studies and is often hampered by methodological issues. To assess the replicability of SCA7 mouse phenotype, a second SCA $7^{140 Q / 5 Q}$ mouse colony was raised in the husbandry of a different research center and was analyzed by independent experimenters and instruments. Mice from the second colony were tested using the standardized protocols established for the first colony (see Methods for further details). As in the first colony, SCA ${ }^{140 Q / 5 Q}$ males gained less weight than WT littermates, reaching a plateau at 14 weeks (Additional file 3: Figure S3a). However, the average weights of adult SCA $7^{140 Q / 5 Q}$ and WT males from the second colony were $6.5 \%(p<0.0001)$ lighter than the mice of the first colony (Additional file 3: Figure S3b), indicating a significant environment effect regardless of the genotype. As for the first colony, open field analysis revealed the hypoactivity of SCA $7^{140 Q / 50}$ mice from 16 weeks of age, with a decreased total distance travelled in the arena, a decreased average speed, an increased resting time and a decreased number of rears (Additional file 3: Figure S3c-f). In contrast to the first colony, the exploration of the open central area (Additional file 3: Figure S3g) suggested a tendency of SCA7 mice to anxiety behavior in the second environment. As for the first colony, SCA $7^{140 Q / 5 Q}$ mice of the second colony took significantly longer time to cross the beam walking bar (Additional file 3: Figure S3h). However, accelerated rotarod, which had a smaller rod diameter than the previous apparatus used for the first colony, led to short latency to fall even for WT at baseline and hence did not reveal any difference in the latency between the two genotypes (Additional file 3: Figure S3i).

In summary, the hypoactivity of SCA $7^{140 Q / 5 Q}$ mice revealed by open field tests appeared at an early time point (onset at 16 weeks), while defects in motor performances (as shown in rotarod of the first colony) and in specific proprioceptive abilities (as reflected by gait alteration in notched bar, beam walking, footprint and catwalk) appeared later between 20-25 weeks, depending of the test. Except for rotarod, the onset and progression of motor phenotypes of $S C A 7^{140 Q / 5 Q}$ were replicable in two different research centers.

\section{Morphological and functional alterations of the peripheral nervous system.}


To understand the cause of motor alterations in SCA $7^{140 Q / 5 Q}$ mice, the activity of the peripheral nervous system was first assessed over time by measuring the sciatic nerve function by electromyography. At 15 weeks, median amplitudes of plantar M-wave and H-wave of SCA $7^{140 Q / 5 Q}$ mice were similar to WT littermates (Fig. 4a-b). However, both plantar M-wave and H-wave amplitudes were reduced by $21 \%$ ( $\mathrm{p}<$ $0.05)$ and by $33 \%(p<0.05)$, respectively, at 32 weeks in SCA7 mice. At 43 weeks, the plantar M-wave amplitude was reduced by $31 \%(p<0.05)$, while the $\mathrm{H}$-wave was almost absent $(95 \%, \mathrm{p}<0.0001)$. To correlate these functional abnormalities with structural status, sciatic nerve sections were analyzed by histology and electron microscopy. Compared to WT mice, cross section of sciatic nerve of SCA7 mice showed clear alterations of myelinated fibers and decreased number of small fibers (Fig. 4c). On electron microscopy (Fig. 4d-o), several structural alterations were observed in myelinated fibers of SCA $7^{140 Q / 5 Q}$ mice, including myelin degeneration and infolding-like structures as well as axonal modifications characterized by inner swelling tongue and autophagy. Non-myelinated fibers displayed abnormal structures of Remak bundles. In conclusion, motor components of the sciatic nerve morphology and activity are affected in SCA $7^{140 Q / 5 Q}$ mice.

\section{Distinct morphological alterations in SCA7 mouse brain.}

Brains of SCA7 patients show prominent neurodegeneration in the cerebellum and atrophy of other brain regions. To establish a systematic survey of brain damage of SCA7 mice, the morphology of three different coronal brain sections of SCA $7^{140 Q / 5 Q}$ and WT mice was studied using neuroanatomical measurement of 78 anatomy parameters across 20 distinct brain subregions (28). At 33 weeks, there was no major surface difference between $S C A 7^{140 Q / 5 Q}$ mice and WT littermates. However, end-stage SCA $7^{140 Q / 5 Q}$ mice (50 weeks) showed significant reduction of brain areas in the three coronal sections (from $-6.7 \%$ to $-8 \%$ ) with both white and grey matter alterations (Additional file 3; Figure S4). Atrophy of grey matter structures included the somatosensory cortex, the pons, while affected white matter regions comprised the corpus callosum, the anterior commissure, the internal capsule and the fimbria of the hippocampus.

We then aimed to capture SCA7 mouse brain alterations at earlier disease stage using high resolution MRI. MRI measurement clearly showed a global atrophy of the SCA7 mouse brains compared to WT at 25 weeks $(p<0.05)$ (Fig. 5a). Moreover, the decrease of whole brain volume of SCA $7^{140 Q / 140 Q}$ mice was two times higher than $S C A 7^{140 Q / 5 Q}(p<0.05)$, confirming the stronger severity of the disease in homozygous mice. Interestingly, our automated segmentation pipeline highlighted specific brain regions particularly atrophied in the mouse model such as the corpus callosum, and subparts of the hippocampus and cortex (Fig. 5b and Additional file 3: Figure S5). No significant atrophy of the cerebellar cortex was measured in SCA7 mice, which can be due to strong variability of volume measurement in this structure.

To further correlate cerebellar neuropathology and motor alterations, morphometric parameters were measured on sagittal sections of the central vermis at different disease stages (Fig. 5c-g). The vermis length (axis from lobule V to IX) of SCA7 ${ }^{140 Q / 5 Q}$ mice was smaller to WT at 34 weeks (Fig. 5c). 
Interestingly, the vermis width (axis from lobule VI to X) was significantly shorter in SCA7 ${ }^{140 Q / 5 Q}$ mice at 12 weeks already and this difference remained at later stages but did not worsen with the motor phenotype (Fig. 5d). Strikingly, there was no major or progressive alteration of the molecular cell layer (MCL) thickness in any of the lobules analyzed (Fig. 5f, $g$ and data not shown).

\section{Morphological and functional alterations of Purkinje cells.}

Since PC are primary targets in SCA7, their morphology, connectivity and function were investigated. The area of PC soma was significantly smaller in lobule VI $(p<0.002)$ of 34 -week SCA7 ${ }^{140 Q / 50}$ compared to age-matched WT mice, while in lobules IX and X PC soma did not differ (Fig. 6a-e). In contrast, PC circularity index was affected in lobules $I X$ and $X(p<0.006$ and $p<0.0005$, respectively), but not in lobule VI (Fig. 6f-h). We then analyzed synapses integrity between PC and climbing fibers (CF) of inferior olivary neurons. To this purpose, we quantified vGLUT2-immunolabeling of CF-PC contact points in the molecular cell layer using the image analysis Imaris software. The average density and average volume of vGLUT2 contact points were similar in WT and SCA7 mice (data not shown). However, extension of climbing fiber terminals along the PC dendritic tree was significantly reduced in lobule VI and X by $15.8 \%$ $(p=0.029)$ and $23 \%(p=0.034)$, respectively, in SCA $7^{140 Q / 5 Q}$ compared to WT mice (Fig. 6i, j). Moreover, coimmunolabeling of VGLUT2 and CALB1 revealed frequent aggregation of vGLUT2 contact points along large dendritic arborization of PC in SCA7 cerebellum (Fig. 6k). Therefore, despite different morphological alterations of PC soma of lobule $\mathrm{VI}$ and $\mathrm{X}$, synaptic contacts between PC and CF were affected in both lobules.

Intrinsic membrane properties of PC determine their spontaneous firing pattern even in the absence of synaptic inputs $(38,39)$. Any alteration in the precision of their pacemaking activity may be the result of PC cellular dysfunctions and may affect cerebellar functions (40). To investigate the correlation between PC function, morphological phenotypes and motor deficits, we assessed whether the intrinsic properties of PC spontaneous discharge were altered in SCA $7^{140 Q / 5 Q}$ mice at 2 disease stages (22 and 38 weeks). We recorded spontaneous activity of PC located in lobule VI and IX/X in WT and SCA7 littermates using juxtacellular recordings of $\mathrm{PC}$ in acute cerebellar slices at a near physiological temperature $\left(32^{\circ} \mathrm{C}\right)$ (Fig. 6l). To focus on PC intrinsic excitability and to rule out network contributions, excitatory and inhibitory synaptic transmissions were pharmacologically blocked. PC discharges were evaluated using the mean firing frequency (measurement of the mean ISI) and the CV2, which estimates the variability of the firing pattern between two consecutive ISIs $(41,42)$. At 22 weeks, no difference in the firing properties of PCs located in lobule VI was detected between WT and SCA7 mice. In contrast, in symptomatic SCA7 mice of 38 weeks, PC exhibited a higher regularity of discharge in both lobules $\mathrm{VI}(\mathrm{p}<0.001)$ and IX/X ( $p<$ 0.001 ) (Fig. $6 \mathrm{~m}, \mathrm{n})$ and a decrease in the mean firing rate in lobule $\mathrm{VI}(\mathrm{p}=0.004)$ (Fig. 60). Our results show that intrinsic properties of PC excitability are altered in symptomatic SCA7 mice.

\section{Accumulation of mutant ATXN7 in retina and cerebellum.}


Studies of SCA7 cellular and animal models showed that mATXN7 tends to misfold and form protein aggregates (reviewed in (16)). Immunofluorescence analysis of control adult retina and cerebellum showed that WT ATXN7 is barely detectable (Fig. 7a and b, left panels). In contrast, the immunolabeling progressively increased with age in SCA7 mouse tissues, indicating that mATXN7 accumulates over time. This resulted in different labeling profiles depending of the neuronal population. In SCA7 retina at 12 weeks, mATXN7 aggregates were readily detected in many photoreceptor nuclei and in some neuronal nuclei of ganglion and inner nuclear layers (Fig. 7a). mATXN7 aggregates became widespread in retinal neuron nuclei at later stages. In SCA7 cerebellum, PC showed the earliest and most important mATXN7 accumulation that progressively increased between 12 and 19 weeks and progressively led to the formation of large nuclear aggregates at later stages (Fig. 7b). mATXN7 nuclear aggregates were also observed in the $\mathrm{MCL}$ and granule cell layer (GCL). All cerebellar lobules showed the accumulation of mATXN7 in PC nuclei (Fig. 7c).

Western blot analysis showed the expression of WT ATXN7 as a 110 kilodalton (kDa) band in control retina and cerebellar samples (Fig. 7d). In SCA7 samples, the mutant form of ATXN7 migrated at 140 kDa. Importantly, mATXN7 was also detected as high molecular weight smear from 5-8 weeks of age, indicating that mATXN7 accumulates as insoluble oligomeric forms in retinal and cerebellar tissues, much before the formation of visible nuclear aggregates as detected by immunolabeling of tissues.

\section{Cerebellar gene expression changes in SCA7 mice.}

To gain molecular insight into the cerebellar dysfunctions of SCA $7^{140 Q / 5 Q}$ mice, gene expression profiles of cerebellar samples of symptomatic SCA7 mice and their WT littermates were analyzed by RNA-seq. Analysis of differentially expressed genes indicated that 406 genes are downregulated in SCA7 cerebella and 270 genes are upregulated $(p<0.05)$ (Fig. 8a). Ingenuity Pathway Analysis (IPA) (36) of the 676 differentially expressed genes revealed that the most significantly affected functions $(p \leq 0.0002)$ concern major neuronal signaling pathways involving glutamate, G-protein coupled receptors, calcium, CREB and CAMP, and LTD (Fig. 8b and Additional file 5: Table S4), most of which have previously been implicated in the pathogenesis of other ataxias (40).

IPA also pointed out the impairment of interferon signaling pathway (Fig. 8b). Interestingly, among the 10 most predicted upstream regulators $\left(p \leq 7 * 10^{-9}\right)$ of the differentially expressed genes, 8 are implicated in the modulation of interferons signaling pathways (Additional file 6: Table S5). Moreover, functional annotations of the 270 upregulated genes using STRING (35) further highlighted a strong enrichment (false discovery rate $(\mathrm{FDR})<2^{*} 10^{-6}$ ) of type 1 interferon pathway (Additional file 3: Figure S6). The interferon regulator factor 7 gene (IIf 7$)$, which is the second most significantly upregulated protein-coding gene ( 2.7 fold; $p=2.28 * 10^{-10}$ ) in SCA7 mouse cerebella, composed with high confidence $(0.700)$ a protein-protein association network of 19 direct edges that includes signal transducer and activator of transcription 1 (Stat1), which further interconnects with the proto-oncogene Jun. Together, Irf7, Stat1 and Jun constituted the center a large network of 189 edges involving multiple upregulated genes coding for proteins involved in immune system response (Additional file 3: Figure S6), further emphasizing the 
important activation of interferon signaling pathway in SCA7 cerebella. Chort et al. (43) previously reported the increased level of interferon-beta and its receptor in the cerebellum of the SCA7 $266 \mathrm{Q} / 5 \mathrm{Q}$ model. Our transcriptome data provide additional evidence of the activation of a regulatory network involving interferon signaling.

\section{Downregulation of a large subset of Purkinje cell-enriched genes.}

To gain insight into the cell type-specific distribution of differentially expressed genes in SCA7 cerebella, we took advantage of the publicly available actively translated mRNA profiles of all the major cerebellar cell types (37). To compare with our SCA7 RNA-seq dataset, the published datasets (37) were reannotated using Affymetrix microarray probesets annotations on mm10 genome assembly. This reannotation led to a total of 9671 actively translated transcripts in the different cell types of WT cerebellum (Additional file 3: Figure S7). $89 \%$ of them were present in the SCA7 dataset. Out of 9671 actively translated transcripts, we identified 5058 transcripts present in only one of the nine major cerebellar cell type datasets, including 801 enriched transcripts in PC, 2357 in granule cells, 156 in Golgi cells, 340 in stellate and basket cells, 144 in unipolar bruch cells, 563 in oligodendrocytes expressing Olig2, 133 in oligodendrocytes expressing Cmtm5, 279 in Bergmann glia and 285 in astrocytes (Additional file 3: Figure S7 and Additional file 7: Table S6). Taking the cellular distribution into account, we were able to determine the cell type enrichment of 470 differentially expressed genes of SCA7 cerebellum and found that 240 of them were enriched in a single cerebellar cell type and 230 were present in more than one cell type (Additional file 8: Table S7). The analysis revealed that all the cerebellar cell types presented differentially expressed genes (Fig. 8c, d), indicating that SCA7 pathology is widespread across cerebellar cell types. However, despite the rarity of PC in the cerebellum estimated to $0.1 \%$ of cerebellar cells, $23 \%$ (106 out of 470 ) of SCA7 deregulated genes were enriched in PC. In contrast, $15 \%$ of SCA7 deregulated genes were enriched in granule cells, while this cell type represents $90 \%$ of cerebellar cells. Importantly, while granule cell-enriched genes were both up- and downregulated, almost all PC-enriched genes (104 out of 106 genes) were downregulated (Fig. 8d), indicating that repression of PC-enriched genes is a major component of SCA7 cerebellar pathology. Another publicly available datasets from Kratz et al. (44) allowed us to identify 5 additional PC-enriched genes that were downregulated in SCA7 mouse cerebella (Additional file 8: Table S7), resulting in a total 109 downregulated PC-enriched genes. To ascertain the enrichment of these 109 genes in PC, their level of expression was analyzed using in situ hybridization datasets reported in The Brain Transcriptome Database (BrainTx; CDT) (45) and in the Allen Brain Atlas (30). We established three categories of genes according to the level of enriched expression in $\mathrm{PC}$, as specifically expressed or strongly enriched in PC compared to other cerebellar cells, enriched in PC or non-enriched in PC (e.g. equally present in other cell types). The analysis showed that 56 genes were specific or strongly enriched in PC, 27 genes had moderate enrichment in PC, while 14 other genes were expressed in wider range of cerebellar cell types and 12 genes were not present in in situ hybridization datasets (Additional file 9: Table S8). In fine, 83 genes had validated enriched expression in PC of WT mice and showed reduced expression in PC of SCA7 mice. 
RT-qPCR was used to study the time course of repression of a subset of PC-enriched genes in SCA7 ${ }^{140 Q / 5 Q}$ cerebella. The Grid2, Calb1, Fam21 and Rora genes showed decreased expression in SCA7 mice from 27 weeks, coinciding with the onset of motor alterations (Additional file 3: Figure S8). Interestingly, the 3 PC-enriched genes Pcp4, Fam107b and Rgs8 showed downregulation already at 12 weeks, a pre-symptomatic stage of the disease, suggesting a strong implication in PC pathology of SCA7 mice.

\section{A signature of downregulated PC-enriched genes in SCA1, SCA2 and SCA7.}

To further explore whether PC-enriched genes downregulated in SCA7 have pathological relevance in cerebellar ataxia, we looked at publicly available transcriptomic datasets of SCA1 and SCA2 mouse models in which the mutant genes were specifically expressed in PC and caused PC pathology $(46,47)$. In these 2 models, expression level of a subset genes was also shown to be altered compared to their respective WT littermates $(46,47)$. Before proceeding with the comparative analysis, we have reanalyzed the RNA-seq raw data of SCA1 mouse cerebella at 5, 12 and 28 weeks and SCA2 cerebella at 6 weeks with the same software which was used for SCA7. We then compared the datasets of differentially expressed genes in three SCA models. The analysis revealed that 136 genes common to the 3 datasets were downregulated and only 5 were upregulated (Fig. 8e and 8f). Interestingly, out of the 136 downregulated genes, 67 were PC-enriched including 10 genes associated with genetic ataxias (Table 1 and Additional file 10: Table S9). These 67 genes were downregulated already at 5 weeks in SCA1 and at 6 weeks in SCA2 mice, which are disease onset stages of these models $(47,48)$, and constitute a signature of early pathological processes of PC. The comparison performed with the SCA1 dataset at later disease stages revealed 13 additional downregulated PC-enriched genes in common with SCA2 and SCA7 mice (Table 1), suggesting that these 13 genes were associated with the progression of PC pathology.

The biological significance of the early downregulation of the 67 PC-enriched genes was researched using 3 analyses. First, the KEGG pathway analysis indicated that this set was enriched for genes involved in LTD (FDR 0.0036), cGMP-PKG signaling pathway (FDR 0.0068) and phosphatidylinositol signaling system (FDR 0.0069) (Fig. 8g). Second, the STRING analysis (35) was used to search for functional partnerships and interactions between proteins encoded by the $67 \mathrm{PC}$ genes. It revealed a network of 45 edges ( $p$ value $=1.2 \mathrm{e}^{-13}$ ) $($ Fig. $8 \mathrm{~g}$ ) interconnecting a large number of proteins encoded by genes showing mutations in human or mouse model ataxias: Grid2, Calb1, Car8, Trpc3, Kcnma1, Inpp5a, Inpp4a. The network also showed central positions to the regulator of G-protein signaling 8 (RGS8), phospholipase $C$ beta 3 (PLCB3) and cGMP-dependent kinase PRKG1, the latter two are related to LTD and synapse functions of PC $(40,49)$. Finally, our list of 67 PC-enriched genes was compared to a published list of genes composing the SCA1 Magenta gene module (47). This module was established using the WGCNA (Weighted Gene Coexpression Network Analysis) of SCA1 cerebellar transcriptomes and reported to be enriched in PC genes involved in LTD and glutamate signaling and to be of high relevance for SCA1 pathogenesis (47). We found that 48 out of 67 PC-enriched genes were present in the Magenta module. Furthermore, two major hub genes described in the module, namely Fam107b and 
Rgs8, were within the top strongest downregulated genes in SCA1, SCA2 and SCA7 models, and were already downregulated at 12 weeks in SCA7 mice, hence before onset of motor symptoms (Additional file 3: Figure S8). Altogether, these analyses suggest that the dysfunction of synaptic LTD of PC constitutes a common pathway in the early pathogenesis of SCA1, SCA2 and SCA7.

Finally, to identify mechanisms which could account for the downregulation of the 67 PC-enriched genes, we first looked at RORa (RAR-related orphan receptor alpha), a key transcription factor involved in PC differentiation and survival (50) and showing downregulation in SCA1, SCA2 and SCA7 mice (Table 1). Among the 67 PC-enriched genes, 19 genes were known RORa-target genes $(50,51)$ (Table 1), suggesting that RORa dysfunction partially accounts for PC genes deregulation. We then performed in silico analysis of 2-kb promoter sequence of the $67 \mathrm{PC}$-enriched genes to search for de novo motifs and transcription factors binding sites using MEME, TOMTOM, AME or FIMO (52). The analysis did not reveal major enrichment of transcription factor binding motifs in the promoter of the 67 PC-enriched genes affected, compared to control sequences (e.g. shuffled promoter sequences or promoter sequences of 67 PCenriched non-deregulated genes) (data not shown). Deregulation of gene expression may depend on alteration of diverse processes including chromatin structure, nucleosomal occupancy and histone modifications, which play important roles in transcriptional regulation. Given the presence of ATXN7 in the histone-modifying SAGA complex, we determined whether the bulk level of histone marks regulated by SAGA were altered in SCA7 cerebellum. The level of H2Bub was significantly increased in SCA7 ${ }^{140 Q / 5 Q}$ cerebellum compared to WT, while the level of H3K9ac showed no significant difference (Fig. 9a, b). However, a significant decrease of $\mathrm{H} 3 \mathrm{~K} 9 \mathrm{ac}$ was observed in $\mathrm{SCA} 7^{140 \mathrm{Q} / 140 \mathrm{Q}}$ homozygous mice, which were more severely affected that the SCA7 heterozygous mice (Additional file 3: Figure S9). Together, this suggests that mutant ATXN7 differentially alters the histone-modifying activities of SAGA complex along the progression of cerebellar pathology and might impact on transcription of cerebellar genes.

\section{Discussion}

SCA7 is a devastating neurological disease and no treatment can currently prevent or slow down the disease process. Accurate models of the disease are required as relevant tools for a better understanding of SCA7 pathogenesis and for developing therapeutic strategies. The SCA $7^{140 Q / 5 Q}$ mouse line reported here exhibits cardinal disease features observed in adult SCA7 patients, including retinal, cerebral, cerebellar and peripheral nerve pathologies. Symptoms develop and progress in a suitable time window for preclinical trials and can be quantitatively evaluated using a series of robust motor, functional, histological and molecular parameters (Fig. 10).

SCA $7^{140 Q / 5 Q}$ mice are derived from the $S C A 7^{226 Q / 5 Q}$ mouse line (26), which was previously generated using a knock-in approach to introduce 266 CAG repeats in the Atxn 7 mouse gene to ensure the correct temporal and spatial expression of different ATXN7 isoforms. In human, CAG expansions ranging from 200-460 repeats cause infantile-onset SCA7, which differs from the adult form to some degree (17-21). The SCA $7^{226 \mathrm{Q} / 5 \mathrm{Q}}$ line parallels infantile SCA7 with onset of ERG and rotarod dysfunctions at 5 weeks and 
a very rapid and severe progression leading to premature death at 16 weeks that may mask some disease features. The SCA $7^{140 Q / 50}$ line recapitulates at lower pace most of SCA7-like phenotypes reported in the SCA $7^{226 Q / 5 Q}$ line, and reveals many additional phenotypic features such as the alteration of the RPE and Bruch's membrane, PC firing and synaptic dysfunction and atrophy of different brain regions including white matter, that were not described for the $S C A 7^{226 Q / 50}$ line, possibly due to the premature death. Finally, using a wide range of tests and methodologies, we largely extend the phenotypic description of knock-in models of SCA7: i) by unraveling a peripheral nerve dysfunction and dysmorphology, ii) by providing a comprehensive time-line of functional, histological and molecular alterations underlying degeneration photoreceptors and PCs, two highly vulnerable neurons in SCA7, and iii) by identifying a signature of transcriptional alterations underlying PC dysfunction. Unlike the SCA $7^{226 \mathrm{Q} / 5 \mathrm{Q}}$ line, SCA $7^{140 Q / 5 Q}$ mice present no complication related to husbandry, as fertility of males was normal and females showed no difficulty to deliver.

\section{Retinal, CNS and PNS features of SCA7 mice.}

The vision and motor dysfunctions of SCA $7^{140 Q / 5 Q}$ mice start at 10 and 16 weeks, respectively, progress over a period of 25 weeks, before the health status strongly declines and mice die at around 54 weeks. As reported in earlier SCA7 mouse models $(16,26,53-55)$, photoreceptor dysfunction in SCA ${ }^{140 Q / 5 Q}$ mice closely correlates with the progressive reduced expression of photoreceptor-specific genes such as opsin genes. In normal condition, sustained expression of photoreceptor genes is essential to the daily renewal of part of outer segments, hence to normal photoreceptor function. In SCA $7^{140 Q / 5 Q}$ mice, the decreased expression of photoreceptor genes progressively leads to disappearance of outer segments, as shown by OCT and electron microscopy. In zebrafish, knock-down of atxn7 impedes the formation of photoreceptor outer segments during development of the eye $(23,24)$. Together, this suggests that mATXN7 toxicity alters a normal function of ATXN7 in the maintenance of outer segments in SCA7 mice (16). Atxn7 knockdown in zebrafish also leads to incomplete formation of RPE and Bruch's membrane of the developing eye (23). Aged SCA $7^{140 Q / 5 Q}$ mice show alteration of these two structures with disorganization of the Bruch's membrane and abnormal infolding membranes of RPE. Interestingly, homogenous deposits are observed between RPE plasma membrane and basement membrane, which resemble sub-RPE deposits reported in a mouse model of Malattia Leventinese (56), a dominantly inherited macular degenerative disease. Malattia Leventinese mouse bears a mutation in the causative Efemp1 gene. Kahle et al (57) reported an interaction between ATXN7 and EFEMP1 using yeast two hybrid, which warrants further investigation of the molecular and functional relationship between the two proteins in the context of macular degeneration.

Alteration of spontaneous motor activities of $S C A 7^{140 Q / 5 Q}$ mice are observed at 16 weeks as measured by open field in two different research centers. Motor incoordination occurs between 20-24 weeks, depending of the motor aptitude tested. At onset of hypoactivity and motor incoordination, the apparent general health status, the body composition and the day and night food intake (data not shown) are normal and 
the muscle strength measured with the grip strength test and normalized by the animal body weight did not reveal major alteration.

Early studies of SCA7 patients reported contradictory results about peripheral nerve involvement $(13,58)$. We provide strong evidence that peripheral pathologies account for the neurological phenotype in $S C A 7^{140 Q / 5 Q}$ mice. Electromyograph studies revealed that motor peripheral activities are reduced at 32 weeks, consistent with lesions of myelin and myelinated fibers of sciatic nerve as observed at ultrastructural level. The sensory component might also be affected as non-myelinated fibers also show alterations. SCA ${ }^{140 Q / 5 Q}$ mice findings parallel recent studies of SCA7 patients, who showed abnormal motor and sensory nerve conductions (12), and complement previous neuropathological evidence of degenerating signs in the dorsal root and anterior horn of patients' spinal cord $(5,7)$. Like SCA7, other polyQ SCA patients are affected by peripheral neuropathy symptoms and peripheral nerve pathology was reported in other polyQ SCA model $(59,60)$. Given the close parallel between patients and mice, anomalies of nerve conduction represent a relevant therapeutic target and evaluation of electrophysiological nerve conduction may constitute a biomarker with objective outcome parameters for preclinical and clinical trials.

Besides the consistent grey and white matter alterations of the cerebellum and associated structures, post mortem patient brains also showed atrophy and neuronal loss in diverse brain regions, which could account for a variety of neurological symptoms present in adult-onset SCA7 patients $(4,5)$. Using different approaches, we show that the SCA $7^{140 Q / 50}$ mouse line presents whole brain atrophy and decreased volume of selected brain regions at 24 weeks. Interestingly, MRI analysis of 24-week-old SCA7 mice show atrophy of the corpus callosum, hippocampus and cortical regions, which parallels observations of human SCA7 $(5,61)$. In particular, recent studies showed altered functional connectivity between patients' cerebellum and hippocampus or parahippocampal areas that may account for specific memory dysfunctions $(8,10,11,62)$. The hippocampus is a brain region where mutant ATXN7 rapidly accumulates in SCA7 ${ }^{140 Q / 5 Q}$ mice (data not shown) and in SCA ${ }^{266 Q / 5 Q}$ mouse line (26). In the latter model, mATXN7 accumulation was correlated to specific alteration of the short-term synaptic plasticity of hippocampal neurons (26). The cerebellar cortex shows no significant atrophy in 24-week-old SCA7 mice, which can be due to strong variability of volume measurement by MRI in this structure. Indeed, in our ex vivo imaging setup, the cerebellum was peripheral in the field of view, where the magnetic field was less homogeneous and coil sensitivity was lower. Further improvements of our automated pipeline and imaging protocol would be necessary to improve the characterization of volume variations in this particular brain region.

Direct morphometric analysis of SCA7 cerebellar sections shows that the vermis width is slightly but significantly smaller before the onset of the motor symptoms. However, we observed no significant thinning of the molecular cell layers, no loss of PC, no decreased Calbindin 1 and no increased GFAP labeling (data not shown), that could explain the gross morphological alterations of cerebellar vermis. Other morphometric parameters of the cerebellum such as loss of other cerebellar cell types or decrease myelination, might account for the vermis alterations and would require further investigations. At end- 
disease stage (52 weeks), SCA $7^{140 Q / 5 Q}$ brain shows additional atrophy of the pons and somatosensory cortex as well as the anterior commissure, the internal capsule and the fimbria of the hippocampus. Overall, brain alterations detected in the SCA $7^{140 Q / 50}$ mice recapitulate many characteristics of human SCA7 and extend previous finding in SCA $7^{226 Q / 5 Q}$ knock-in.

\section{PC pathology of SCA7 mice.}

Despite the marked degeneration of PC in the post-mortem cerebellum of SCA7 patients (3-5), little is known about the mechanism underlying PC pathology. Our study of SCA $7^{140 Q / 5 Q}$ mice indicates that PCs show morphological alterations of soma and synapses, impairment of spontaneous firing and loss of expression of many genes involved firing and synaptic functions, but no overt PC loss, indicating that PC dysfunction largely precedes cell demise.

It is well known that precision of PC pacemaking activity is essential for normal cerebellar functions and any impairment of PC intrinsic excitability leads to ataxic behaviors (40). In several rodent models of ataxia, PCs are characterized by a lower rate of spontaneous firing compared to WT and/or by an irregular firing pattern $(40,48,63-65)$. Here, we show that in SCA7 mice at 38 weeks, PCs fire with a higher regularity in lobules $\mathrm{VI}$ and $\mathrm{IX} / \mathrm{X}$ and a lower frequency in lobule $\mathrm{VI}$, compared to WT littermates.

Properties of $\mathrm{PC}$ pacemaking activity are shaped by a complex interplay between $\mathrm{Ca}^{2+}, \mathrm{Na}^{+}$and $\mathrm{K}^{+}$ conductance (66) and mutations or deletions of genes encoding for these ion channels are causal factors of many types of ataxia (40). In SCA7 knock-in mice, expression of several PC genes associated with potassium channels is downregulated (Table 1 ) and may explain the alterations of PC pacemaking activity (67). This hypothesis is reinforced by the fact that in SCA7 mice PC exhibits a higher regularity of their firing pattern (low CV2), a phenotype already observed in ataxic waddles mice (68). Interestingly, in waddles mice, mutation in Car8 gene leads to low CV2 of PC firing and marked ataxia syndrome in the absence of any alteration of the PC and molecular cell layers, as in SCA7 mouse cerebellum. Car8 gene is one of the 83 PC genes downregulated in SCA7 mice. Interestingly, in waddles mice, the low CV2 of PC activity has been restored to regular values by chlorzoxazone, a pharmacological agent increasing the probability of opening of $\mathrm{Ca}^{2+}$-dependent $\mathrm{K}^{+}$channels (68). The PC firing impairment of SCA7 ${ }^{140 \mathrm{Q} / 5 \mathrm{Q}}$ mice differs from the PC excitability alterations recently reported in the transgenic fXSCA7 92Q mice, which overexpress the human ATXN7 CDNA inserted in the murine prion gene in a bacterial artificial chromosome (69). PCs of fxSCA7 92Q mice exhibited normal spiking frequency but increased coefficient of variation (CV) of firing (69). The difference between the pattern of PC firing observed in SCA7 ${ }^{140 Q / 5 Q}$ and fxSCA7 92Q mice may result from difference in the cerebellar location of the recorded PCs as PC microcircuits are non-uniform in the cerebellar cortex or in the disease severity of the recorded PCs. Other explanations might come from difference in the number of PC-enriched downregulated genes between the two models (31 PC genes in fxSCA7 92Q mice versus 83 PC genes in SCA7 ${ }^{140 Q / 5 Q}$ mice), and in model designs (transgenic versus knock-in mice; human cDNA encoding ATXN7a isoform versus endogenous mouse gene; overexpression versus endogenous expression of mutant ATXN7 $(26,69)$ ). 
Whatever the factors underlying impairment of PC excitability in SCA $7^{140 Q / 5 Q}$ mice, they differentially affect PCs in lobule VI and in lobule IX/X, since a slowing-down of the firing frequency is only observed for PCs located in lobule VI. We also observed different morphological alteration of PCs in lobule VI (smaller soma) and in lobule IX/X (altered circularity) that may be linked to their excitability impairment. In WT mice, intrinsic excitability is heterogeneous among PC (70) and our recordings in WT mice did show differences in the firing frequency between PCs located in lobule VI and PC located in lobules IX/X. Based on the topographical organization of the olivo-cortico-cerebellar network (71), lobule-specific differences in PC firing rate between WT and SCA7 may suggest that defects are associated to specific cerebellar tasks.

Synaptic functions integrating afferent signals that arrive from CF, parallel fibers (PF) or interneurons are essential to control the output of PCs and mutations affecting the function of synaptic circuits lead to ataxia (40). SCA7 ${ }^{140 Q / 50}$ dysfunction at CF-PC synapses is revealed by the reduced vGLUT2 immunolabeling in the molecular cell layer, whereas downregulation of PC-enriched genes encoding postsynaptic membrane-associated proteins such as GLUR62 (coded by Grid2), GRID2ip, GABBR1 and TRPC3 indicates functional alteration of PF-PC synapses. All four proteins are closely related to mGluR1 function, glutamate signaling and LTD (72-75). They are part of the 67 PC-enriched genes commonly downregulated in SCA1, SCA2 and SCA7 (Table 1). Importantly, these 67 PC-enriched genes are downregulated in early symptomatic SCA1 (5 weeks) and SCA2 (6 weeks) $(47,48)$, suggesting that they are primary targets of the pathogenesis in the three SCAs. There is strong interest in identifying converging pathways of PC pathology across SCAs to improve our understanding of polyQ pathogenesis and to identify common potential targets for therapeutic intervention (76). STRING network analysis of the 67 PC-enriched genes further points out that LTD, cGMP-PKG signaling and phosphatidylinositol signaling pathways are commonly altered in SCA1, SCA2 and SCA7. Furthermore, STRING analysis pointed out two hub proteins within the network, PLCB3 and PRKGI, for which the corresponding gene downregulation in SCAs may account for LTD impairment. In a proposed model of LTD $(75,77)$, glutamate signaling at PF-PC and CF-PC synapses activates PLCBs, which catalyze the production of secondary messengers, diacylglycerol (DAG) and inositol 1,4,5-triphosphate (IP3) from phosphatidylinositol 4,5-bisphosphate (PIP2). DAG and IP3 in turn signal, respectively, to PKC and IP3R to successively trigger internalization of AMPARs (a-amino-3-hydroxy-5-methyl-4-isoxazolepropionic acid receptors), synaptic desensitization, and hence LTD. The reduced expression of PIcb3 as well as Itpr1 (IP3R) in SCA1, SCA2 and SCA7 would thus account for the deregulation of $\mathrm{Ca}^{2+}$ signaling and LTD. Interestingly, PLCB3 and IP3R are phosphorylation targets of PRKG1 (78). Deletion of Prkg1 in PC leads to a severe decrease of LTD in PC synapses (49) and mutant mice exhibit an impaired adaptation of the vestibulo-ocular reflex, a function altered in several SCAs (79). It is noteworthy that Plcb3 expression is restricted to the Zebrin II positive parasagittal stripes of the cerebellar cortex (75), and that P/cb3 downregulation in SCA1, SCA2, and SCA7 suggests that PCs located in Zebrin II positive (ZII+) stripes are particularly vulnerable. The dysfunction of ZII+ PC subclass in our SCA $7^{140 Q / 5 Q}$ mice is further supported by the decreased expression of the Zebrin //gene itself (or Aldolase $C, A L D O C$ ) and by firing impairment of PCs located in lobule VI and IX/X of the vermis, which are enriched in ZII+ PCs. 
The molecular mechanisms leading to downregulation of the same subset of PC genes in SCA1, SCA2, and SCA7 remain to be determined. Although ATXN1, ATXN2 and ATXN7 play different roles in cellular mechanisms controlling gene expression (e.g. ATXN1 and ATXN7 are part of transcriptional regulator complexes and ATXN2 functions in RNA homeostasis), there is yet no known functional relationship between the three proteins. We identified 19 PC-enriched genes deregulated in SCA1, SCA2 and SCA7, as known targets of the RORa transcription factor. Interestingly, RORa transactivation activity was shown to be repressed by mATXN7 in cultured cells (80) and the RORa protein itself forms a complex with ATXN1 and TIP60 (81), hence interconnecting possible RORa dysfunction with both mutant ATXN1 and ATXN7 toxicity. In addition, Rora mRNA expression was decreased at pre-symptomatic stage in SCA2 (6 weeks) and at symptomatic stage in SCA1 (12 weeks) and SCA7 (27 weeks) mice. In SCA1 mice, partial loss of TIP60 led to an increase of RORa level and delayed cerebellar pathology (82), further supporting that RORa dysfunction is part of the downstream pathway leading to PC pathology of SCAs.

SCA7 ${ }^{140 Q / 5 Q}$ cerebella show an increase in the bulk level of H2B monoubiquitination, while the level of $\mathrm{H} 3 \mathrm{~K} 9$ acetylation is altered only in the more severely affected SCA $7^{140 Q / 140 Q}$ mice. Similar results were reported in cultured astrocytes expressing mATXN7, which had increased level of H2B monoubiquitination and unchanged level of $\mathrm{H} 3 \mathrm{~K} 9$ acetylation, compared to astrocytes expressing the WT ATXN7 $(83,84)$. These data suggest that mATXN7 differentially alters the histone-modifying activities of SAGA complex. In normal conditions, ATXN7 is a component of the deubiquitination module (DUB) with ATXN7L3, USP22 and ENYA, and anchors DUB to SAGA core complex that contains the histone acetyl transferase (HAT) module. In SCA7 mice, given the strong accumulation of mATXN7 in cerebellar cells, components of DUB might be sequestered in mATXN7 oligomers/aggregates, causing DUB dysfunction, as it was previously shown in cultured cells (84), Alternatively, the unchanged level of H3K9 acetylation might be explained by functional compensation by other HAT-containing complexes such as ATAC present in the cerebellum. Whatever the mechanism, it appears that despite the alteration of the global level of $\mathrm{H} 2 \mathrm{~B}$ monoubiquitination, the expression of only a small number of genes is altered in SCA $7^{140 Q / 5 Q}$ cerebellum. Whether transcript buffering accounts to mitigate gene deregulation in SCA7 mice remains to be investigated.

\section{Conclusions}

The new SCA $7^{140 Q / 5 Q}$ mice represent a promising model to study different degenerative and developmental components of the disease in different tissues and, it offers opportunities for translational development of therapeutic strategies by targeting the brain, retina, peripheral nerve or whole body. Our data also provide insight into converging mechanisms of PC degeneration in polyglutamine SCAs and point out molecular targets for therapeutic development which may prove beneficial for several SCAs.

\section{Abbreviations}


Spinocerebellar ataxia type 7 (SCA7); Purkinje cells (PC); polyglutamine (polyQ); ataxin-7 (ATXN7); mutant ATXN7 (mATXN7); Spt-Ada-Gcn5 Acetyltransferase (SAGA); long-term depression (LTD); optical coherence tomography (OCT); electroretinography activity (ERG); outer nuclear layer (ONL); inner nuclear layer (INL); inner plexiform layer (IPL); green cone opsin (OPN1MW); blue cone opsin (OPN1SW); rhodopsin (RHO); Quantitative RT-PCR (RT-qPCR); retinal pigmented epithelium (RPE); magnetic resonance imaging (MRI); false discovery rate (FDR); wild type (WT); heterozygous (HTZ); homozygous $(\mathrm{HMZ})$; males (M); females (F); Purkinje cell layer (PCL); granular cell layer (GCL); molecular cell layer (MCL); inter-spike interval (ISI); coefficient of variation for adjacent ISIs (CV2); acetylated lysine 9 of histone H3 (H3K9ac); monoubiquitinated histone H2B (H2Bub); Climbing fibers (CF); 4',6-diamidino-2phenylindole dihydrochloride (DAPI); Paraformaldehyde (PFA); Weighted Gene Coexpression Network Analysis (WGCNA); diacylglycerol (DAG); inositol 1,4,5-triphosphate (IP3); phosphatidylinositol 4,5bisphosphate (PIP2); a-amino-3-hydroxy-5-methyl-4-isoxazolepropionic acid receptors (AMPARs); deubiquitination module (DUB); histone acetyltransferase (HAT).

\section{Declarations}

\section{Ethics approval and consent to participate.}

All animal procedures were carried out in strict accordance with the French and German national laws for laboratory animal welfare and the guidelines of the Federation of European Laboratory Animal Science Associations, based on European Union Legislation (Directive 2010/63/EU). Experiments were approved by local Ethics Committees under the supervision of the Ministry of Higher Education, Research and Innovation in France (agreement numbers: \#14199-2018032117483097, \#1803-2015072215481972 and \#4354-2016030212155187-v3; CREMEAS; CEEA35) and the Regierungspraesidium Tuebingen in Germany.

\section{Consent for publication.}

Not applicable.

\section{Availability of data and materials}

The RNA-seq data have been submitted to the NCBI Gene Expression Omnibus (GEO) (http://www.ncbi.nlm.nih.gov/geo/) under GSE138527 (Link https://www.ncbi.nlm.nih.gov/geo/query/acc.cgi?acc=GSE138527 Token for reviewers: slknackkxdwfjaj). Other datasets used and/or analyzed during the current study are available from the corresponding author on reasonable request.

\section{Conflict of interest statement.}

The authors declare no conflict of interest.

\section{Funding.}


This Project has received funding from the EU Joint Programme-Neurodegenerative Disease Research (JPND) Project ModelPolyQ (Grant Agreement No. 643417) under the ANR grant agreement ANR-15JPWG-0008-03 to Y.T. and under the BMBF grant agreement 01ED1606 to H.H.P.N.; from the French foundation Connaître les Syndromes Cérébelleux (CSC) to Y.T.; from ANR-CE37-2015 (CeMod) and Fondation pour la Recherche Médicale (Equipes FRM DEQ20140329514) to P.I.; from ANR-10-LABX-0030INRT under the frame programme Investissements d'Avenir labelled ANR-10-IDEX-0002-02 (to IGBMC). The 11.7T MRI scanner was funded by a grant from NeurATRIS: A Translational Research Infrastructure for Biotherapies in Neurosciences ("Investissements d'Avenir", ANR-11-INBS-0011).

\section{Author's contributions.}

ANC, EB, JF, PI, HHHN and YT conceived the project. Researchers that performed experiments and/or analyzed data: mouse behaviors (ANC, HM, AN, CW, AK, HHHN and AE), retina analyses (MJR), electromyography (FP), morphometry (ANC and BY), electrophysiology of Purkinje cells (FDand $\mathrm{PI}), \mathrm{MRI}$ (JBP, JF and EB), tissue analysis and imaging (ANC, NM, MFC, AH and EG), molecular analysis (ANC, CK and $A H)$. ANC and YT supervised the project and wrote the first draft of the manuscript. All authors read, edited and approved the manuscript.

\section{Acknowledgments.}

We thank Dr Huda Zoghbi for providing the SCA7 knock-in mice, the Mouse Clinical Institute of Illkirch for mouse phenotyping support, the GenomEast Platform at IGBMC, a member of the 'France Genomique' consortium (ANR-10-INBS-0009) for processing Illumina libraries, Stephan Collins for macro allowing PC morphological analysis, Filiz Kavla, Raphaël Bour and Christel Wagner for technical assistance, Tao Ye for fruitful discussion regarding motif enrichments, Doulaye Dembele for suggestions regarding statistical analysis. Special thanks to Nathalie Daigle and Hélène Puccio for critical reading of the manuscript and for fruitful discussion.

\section{References}

1. David G, Durr A, Stevanin G, Cancel G, Abbas N, Benomar A, et al. Molecular and clinical correlations in autosomal dominant cerebellar ataxia with progressive macular dystrophy (SCA7). Human molecular genetics. 1998;7(2):165-70.

2. Martin JJ. Spinocerebellar ataxia type 7. Handb Clin Neurol. 2012;103:475-91.

3. Michalik A, Martin JJ, Van Broeckhoven C. Spinocerebellar ataxia type 7 associated with pigmentary retinal dystrophy. Eur J Hum Genet. 2004;12(1):2-15.

4. Rub U, Brunt ER, Seidel K, Gierga K, Mooy CM, Kettner M, et al. Spinocerebellar ataxia type 7 (SCA7): widespread brain damage in an adult-onset patient with progressive visual impairments in comparison with an adult-onset patient without visual impairments. Neuropathol Appl Neurobiol. 2008;34(2):155-68. 
5. Horton LC, Frosch MP, Vangel MG, Weigel-DiFranco C, Berson EL, Schmahmann JD. Spinocerebellar ataxia type 7: clinical course, phenotype-genotype correlations, and neuropathology. Cerebellum. 2013;12(2):176-93.

6. Martin JJ, Van Regemorter N, Krols L, Brucher JM, de Barsy T, Szliwowski H, et al. On an autosomal dominant form of retinal-cerebellar degeneration: an autopsy study of five patients in one family. Acta Neuropathol (Berl). 1994;88(4):277-86.

7. Martin J, Van Regemorter N, Del-Favero J, Lofgren A, Van Broeckhoven C. Spinocerebellar ataxia type 7 (SCA7) - correlations between phenotype and genotype in one large Belgian family. Journal of the neurological sciences. 1999;168(1):37-46.

8. Alcauter S, Barrios FA, Diaz R, Fernandez-Ruiz J. Gray and white matter alterations in spinocerebellar ataxia type 7: an in vivo DTI and VBM study. Neuroimage. 2011;55(1):1-7.

9. Bang OY, Lee PH, Kim SY, Kim HJ, Huh K. Pontine atrophy precedes cerebellar degeneration in spinocerebellar ataxia 7: MRI-based volumetric analysis. J Neurol Neurosurg Psychiatry. 2004;75(10):1452-6.

10. Hernandez-Castillo CR, Galvez V, Diaz R, Fernandez-Ruiz J. Specific cerebellar and cortical degeneration correlates with ataxia severity in spinocerebellar ataxia type 7. Brain Imaging Behav. 2016;10(1):252-7.

11. Chirino A, Hernandez-Castillo CR, Galvez V, Contreras A, Diaz R, Beltran-Parrazal L, et al. Motor and cognitive impairments in spinocerebellar ataxia type 7 and its correlations with cortical volumes. The European journal of neuroscience. 2018;48(10):3199-211.

12. Salas-Vargas J, Mancera-Gervacio J, Velazquez-Perez L, Rodrigez-Labrada R, Martinez-Cruz E, Magana JJ, et al. Spinocerebellar ataxia type 7: a neurodegenerative disorder with peripheral neuropathy. Eur Neurol. 2015;73(3-4):173-8.

13. van de Warrenburg BP, Notermans NC, Schelhaas HJ, van Alfen N, Sinke RJ, Knoers NV, et al. Peripheral nerve involvement in spinocerebellar ataxias. Arch Neurol. 2004;61(2):257-61.

14. Aleman TS, Cideciyan AV, Volpe NJ, Stevanin G, Brice A, Jacobson SG. Spinocerebellar ataxia type 7 (SCA7) shows a cone-rod dystrophy phenotype. Exp Eye Res. 2002;74(6):737-45.

15. David G, Abbas N, Stevanin G, Durr A, Yvert G, Cancel G, et al. Cloning of the SCA7 gene reveals a highly unstable CAG repeat expansion. Nature genetics. 1997;17(1):65-70.

16. Niewiadomska-Cimicka A, Trottier Y. Molecular Targets and Therapeutic Strategies in Spinocerebellar Ataxia Type 7. Neurotherapeutics. 2019;16(4):1074-96.

17. Benton CS, de Silva R, Rutledge SL, Bohlega S, Ashizawa T, Zoghbi HY. Molecular and clinical studies in SCA-7 define a broad clinical spectrum and the infantile phenotype. Neurology. 1998;51(4):1081-6.

18. Whitney A, Lim M, Kanabar D, Lin JP. Massive SCA7 expansion detected in a 7-month-old male with hypotonia, cardiomegaly, and renal compromise. Dev Med Child Neurol. 2007;49(2):140-3.

19. Ansorge O, Giunti P, Michalik A, Van Broeckhoven C, Harding B, Wood N, et al. Ataxin-7 aggregation and ubiquitination in infantile SCA7 with 180 CAG repeats. Annals of neurology. 2004;56(3):448-52. 
20. Trang H, Stanley SY, Thorner P, Faghfoury H, Schulze A, Hawkins C, et al. Massive CAG Repeat Expansion and Somatic Instability in Maternally Transmitted Infantile Spinocerebellar Ataxia Type 7. JAMA neurology. 2014.

21. van de Warrenburg BP, Frenken CW, Ausems MG, Kleefstra T, Sinke RJ, Knoers NV, et al. Striking anticipation in spinocerebellar ataxia type 7: the infantile phenotype. Journal of neurology. 2001;248(10):911-4.

22. Helmlinger D, Hardy S, Sasorith S, Klein F, Robert F, Weber C, et al. Ataxin-7 is a subunit of GCN5 histone acetyltransferase-containing complexes. Human molecular genetics. 2004;13(12):1257-65.

23. Carrillo-Rosas S, Weber C, Fievet L, Messaddeq N, Karam A, Trottier Y. Loss of zebrafish Ataxin-7, a SAGA subunit responsible for SCA7 retinopathy, causes ocular coloboma and malformation of photoreceptors. Human molecular genetics. 2018.

24. Yanicostas C, Barbieri E, Hibi M, Brice A, Stevanin G, Soussi-Yanicostas N. Requirement for zebrafish ataxin-7 in differentiation of photoreceptors and cerebellar neurons. PloS one. 2012;7(11):e50705.

25. Karam A, Trottier Y. Molecular Mechanisms and Therapeutic Strategies in Spinocerebellar Ataxia Type 7. Advances in experimental medicine and biology. 2018;1049:197-218.

26. Yoo SY, Pennesi ME, Weeber EJ, Xu B, Atkinson R, Chen S, et al. SCA7 knockin mice model human SCA7 and reveal gradual accumulation of mutant ataxin-7 in neurons and abnormalities in shortterm plasticity. Neuron. 2003;37(3):383-401.

27. Chen YC, Gatchel JR, Lewis RW, Mao CA, Grant PA, Zoghbi HY, et al. Gcn5 loss-of-function accelerates cerebellar and retinal degeneration in a SCA7 mouse model. Human molecular genetics. 2012;21(2):394-405.

28. Mikhaleva A, Kannan M, Wagner C, Yalcin B. Histomorphological Phenotyping of the Adult Mouse Brain. Curr Protoc Mouse Biol. 2016;6(3):307-32.

29. Dhenain M, Delatour B, Walczak C, Volk A. Passive staining: a novel ex vivo MRI protocol to detect amyloid deposits in mouse models of Alzheimer's disease. Magn Reson Med. 2006;55(3):687-93.

30. Lein ES, Hawrylycz MJ, Ao N, Ayres M, Bensinger A, Bernard A, et al. Genome-wide atlas of gene expression in the adult mouse brain. Nature. 2007;445(7124):168-76.

31. Yefimova MG, Messaddeq N, Karam A, Jacquard C, Weber C, Jonet L, et al. Polyglutamine toxicity induces rod photoreceptor division, morphological transformation or death in Spinocerebellar ataxia 7 mouse retina. Neurobiology of disease. 2010;40(1):311-24.

32. Kim D, Pertea G, Trapnell C, Pimentel H, Kelley R, Salzberg SL. TopHat2: accurate alignment of transcriptomes in the presence of insertions, deletions and gene fusions. Genome Biol. 2013;14(4):R36.

33. Anders S, PyI PT, Huber W. HTSeq--a Python framework to work with high-throughput sequencing data. Bioinformatics. 2015;31(2):166-9.

34. Love MI, Huber W, Anders S. Moderated estimation of fold change and dispersion for RNA-seq data with DESeq2. Genome Biol. 2014;15(12):550. 
35. Szklarczyk D, Franceschini A, Wyder S, Forslund K, Heller D, Huerta-Cepas J, et al. STRING v10: protein-protein interaction networks, integrated over the tree of life. Nucleic Acids Res. 2015;43(Database issue):D447-52.

36. Kramer A, Green J, Pollard J, Jr., Tugendreich S. Causal analysis approaches in Ingenuity Pathway Analysis. Bioinformatics. 2014;30(4):523-30.

37. Doyle JP, Dougherty JD, Heiman M, Schmidt EF, Stevens TR, Ma G, et al. Application of a translational profiling approach for the comparative analysis of CNS cell types. Cell. 2008;135(4):749-62.

38. Gruol DL, Franklin CL. Morphological and physiological differentiation of Purkinje neurons in cultures of rat cerebellum. The Journal of neuroscience : the official journal of the Society for Neuroscience. 1987;7(5):1271-93.

39. Hausser M, Clark BA. Tonic synaptic inhibition modulates neuronal output pattern and spatiotemporal synaptic integration. Neuron. 1997;19(3):665-78.

40. Hoxha E, Balbo I, Miniaci MC, Tempia F. Purkinje Cell Signaling Deficits in Animal Models of Ataxia. Front Synaptic Neurosci. 2018;10:6.

41. Arancillo M, White JJ, Lin T, Stay TL, Sillitoe RV. In vivo analysis of Purkinje cell firing properties during postnatal mouse development. J Neurophysiol. 2015;113(2):578-91.

42. Holt GR, Softky WR, Koch C, Douglas RJ. Comparison of discharge variability in vitro and in vivo in cat visual cortex neurons. J Neurophysiol. 1996;75(5):1806-14.

43. Chort A, Alves S, Marinello M, Dufresnois B, Dornbierer JG, Tesson C, et al. Interferon beta induces clearance of mutant ataxin 7 and improves locomotion in SCA7 knock-in mice. Brain : a journal of neurology. 2013.

44. Kratz A, Beguin P, Kaneko M, Chimura T, Suzuki AM, Matsunaga A, et al. Digital expression profiling of the compartmentalized translatome of Purkinje neurons. Genome research. 2014;24(8):1396-410.

45. Sato A, Sekine Y, Saruta C, Nishibe H, Morita N, Sato Y, et al. Cerebellar development transcriptome database (CDT-DB): profiling of spatio-temporal gene expression during the postnatal development of mouse cerebellum. Neural Netw. 2008;21(8):1056-69.

46. Dansithong W, Paul S, Figueroa KP, Rinehart MD, Wiest S, Pflieger LT, et al. Ataxin-2 regulates RGS8 translation in a new BAC-SCA2 transgenic mouse model. PLoS genetics. 2015;11(4):e1005182.

47. Ingram M, Wozniak EAL, Duvick L, Yang R, Bergmann P, Carson R, et al. Cerebellar Transcriptome Profiles of ATXN1 Transgenic Mice Reveal SCA1 Disease Progression and Protection Pathways. Neuron. 2016;89(6):1194-207.

48. Hansen ST, Meera P, Otis TS, Pulst SM. Changes in Purkinje cell firing and gene expression precede behavioral pathology in a mouse model of SCA2. Human molecular genetics. 2013;22(2):271-83.

49. Feil R, Hartmann J, Luo C, Wolfsgruber W, Schilling K, Feil S, et al. Impairment of LTD and cerebellar learning by Purkinje cell-specific ablation of cGMP-dependent protein kinase I. The Journal of cell biology. 2003;163(2):295-302. 
50. Gold DA, Baek SH, Schork NJ, Rose DW, Larsen DD, Sachs BD, et al. RORalpha coordinates reciprocal signaling in cerebellar development through sonic hedgehog and calcium-dependent pathways. Neuron. 2003;40(6):1119-31.

51. Sarachana T, Hu VW. Genome-wide identification of transcriptional targets of RORA reveals direct regulation of multiple genes associated with autism spectrum disorder. Molecular autism. 2013;4(1):14.

52. Bailey TL, Boden M, Buske FA, Frith M, Grant CE, Clementi L, et al. MEME SUITE: tools for motif discovery and searching. Nucleic Acids Res. 2009;37(Web Server issue):W202-8.

53. Yvert G, Lindenberg KS, Picaud S, Landwehrmeyer GB, Sahel JA, Mandel JL. Expanded polyglutamines induce neurodegeneration and trans-neuronal alterations in cerebellum and retina of SCA7 transgenic mice. Human molecular genetics. 2000;9(17):2491-506.

54. Abou-Sleymane G, Chalmel F, Helmlinger D, Lardenois A, Thibault C, Weber C, et al. Polyglutamine expansion causes neurodegeneration by altering the neuronal differentiation program. Human molecular genetics. 2006;15(5):691-703.

55. La Spada AR, Fu YH, Sopher BL, Libby RT, Wang X, Li LY, et al. Polyglutamine-expanded ataxin-7 antagonizes CRX function and induces cone-rod dystrophy in a mouse model of SCA7. Neuron. 2001;31(6):913-27.

56. Marmorstein LY, McLaughlin PJ, Peachey NS, Sasaki T, Marmorstein AD. Formation and progression of sub-retinal pigment epithelium deposits in Efemp1 mutation knock-in mice: a model for the early pathogenic course of macular degeneration. Human molecular genetics. 2007;16(20):2423-32.

57. Kahle JJ, Gulbahce N, Shaw CA, Lim J, Hill DE, Barabasi AL, et al. Comparison of an expanded ataxia interactome with patient medical records reveals a relationship between macular degeneration and ataxia. Human molecular genetics. 2011;20(3):510-27.

58. Kubis N, Durr A, Gugenheim M, Chneiweiss H, Mazzetti P, Brice A, et al. Polyneuropathy in autosomal dominant cerebellar ataxias: phenotype-genotype correlation. Muscle Nerve. 1999;22(6):712-7.

59. Liang L, Chen T, Wu Y. The electrophysiology of spinocerebellar ataxias. Neurophysiol Clin. 2016;46(1):27-34.

60. Orengo JP, van der Heijden ME, Hao S, Tang J, Orr HT, Zoghbi HY. Motor neuron degeneration correlates with respiratory dysfunction in SCA1. Dis Model Mech. 2018;11(2).

61. Adanyeguh IM, Perlbarg V, Henry PG, Rinaldi D, Petit E, Valabregue R, et al. Autosomal dominant cerebellar ataxias: Imaging biomarkers with high effect sizes. Neuroimage Clin. 2018;19:858-67.

62. Hernandez-Castillo CR, Alcauter S, Galvez V, Barrios FA, Yescas P, Ochoa A, et al. Disruption of visual and motor connectivity in spinocerebellar ataxia type 7. Mov Disord. 2013;28(12):1708-16.

63. Dorgans K, Salvi J, Bertaso F, Bernard L, Lory P, Doussau F, et al. Characterization of the dominant inheritance mechanism of Episodic Ataxia type 2. Neurobiology of disease. 2017;106:110-23.

64. Stahl JS, Thumser ZC. Flocculus Purkinje cell signals in mouse Cacna1a calcium channel mutants of escalating severity: an investigation of the role of firing irregularity in ataxia. J Neurophysiol. 2014;112(10):2647-63. 
65. Stefely JA, Licitra F, Laredj L, Reidenbach AG, Kemmerer ZA, Grangeray A, et al. Cerebellar Ataxia and Coenzyme Q Deficiency through Loss of Unorthodox Kinase Activity. Molecular cell. 2016;63(4):60820.

66. Raman IM, Bean BP. Ionic currents underlying spontaneous action potentials in isolated cerebellar Purkinje neurons. The Journal of neuroscience : the official journal of the Society for Neuroscience. 1999;19(5):1663-74.

67. Bushart DD, Shakkottai VG. Ion channel dysfunction in cerebellar ataxia. Neurosci Lett. 2019;688:418.

68. White JJ, Arancillo M, King A, Lin T, Miterko LN, Gebre SA, et al. Pathogenesis of severe ataxia and tremor without the typical signs of neurodegeneration. Neurobiology of disease. 2016;86:86-98.

69. Stoyas CA, Bushart DD, Switonski PM, Ward JM, Alaghatta A, Tang MB, et al. Nicotinamide PathwayDependent Sirt1 Activation Restores Calcium Homeostasis to Achieve Neuroprotection in Spinocerebellar Ataxia Type 7. Neuron. 2019.

70. Zhou H, Lin Z, Voges K, Ju C, Gao Z, Bosman LW, et al. Cerebellar modules operate at different frequencies. Elife. 2014;3:e02536.

71. Apps R, Hawkes R. Cerebellar cortical organization: a one-map hypothesis. Nat Rev Neurosci. 2009;10(9):670-81.

72. Kashiwabuchi N, Ikeda K, Araki K, Hirano T, Shibuki K, Takayama C, et al. Impairment of motor coordination, Purkinje cell synapse formation, and cerebellar long-term depression in GluR delta 2 mutant mice. Cell. 1995;81(2):245-52.

73. Kato AS, Knierman MD, Siuda ER, Isaac JT, Nisenbaum ES, Bredt DS. Glutamate receptor delta2 associates with metabotropic glutamate receptor 1 (mGluR1), protein kinase Cgamma, and canonical transient receptor potential 3 and regulates mGluR1-mediated synaptic transmission in cerebellar Purkinje neurons. The Journal of neuroscience : the official journal of the Society for Neuroscience. 2012;32(44):15296-308.

74. Tabata T, Araishi K, Hashimoto K, Hashimotodani Y, van der Putten H, Bettler B, et al. Ca2+ activity at GABAB receptors constitutively promotes metabotropic glutamate signaling in the absence of GABA. Proceedings of the National Academy of Sciences of the United States of America. 2004;101(48):16952-7.

75. Hawkes R. Purkinje cell stripes and long-term depression at the parallel fiber-Purkinje cell synapse. Front Syst Neurosci. 2014;8:41.

76. Smeets CJ, Verbeek DS. Cerebellar ataxia and functional genomics: Identifying the routes to cerebellar neurodegeneration. Biochim Biophys Acta. 2014;1842(10):2030-8.

77. Ito M. The molecular organization of cerebellar long-term depression. Nat Rev Neurosci. 2002;3(11):896-902.

78. Francis SH, Busch JL, Corbin JD, Sibley D. cGMP-dependent protein kinases and cGMP phosphodiesterases in nitric oxide and cGMP action. Pharmacol Rev. 2010;62(3):525-63. 
79. Parker JL, Santiago M. Oculomotor aspects of the hereditary cerebellar ataxias. Handb Clin Neurol. 2012;103:63-83.

80. Strom AL, Forsgren L, Holmberg M. A role for both wild-type and expanded ataxin-7 in transcriptional regulation. Neurobiology of disease. 2005;20(3):646-55.

81. Serra HG, Duvick L, Zu T, Carlson K, Stevens S, Jorgensen N, et al. RORalpha-mediated Purkinje cell development determines disease severity in adult SCA1 mice. Cell. 2006;127(4):697-708.

82. Gehrking KM, Andresen JM, Duvick L, Lough J, Zoghbi HY, Orr HT. Partial loss of Tip60 slows midstage neurodegeneration in a spinocerebellar ataxia type 1 (SCA1) mouse model. Human molecular genetics. 2011;20(11):2204-12.

83. McCullough SD, Xu X, Dent SY, Bekiranov S, Roeder RG, Grant PA. Reelin is a target of polyglutamine expanded ataxin-7 in human spinocerebellar ataxia type 7 (SCA7) astrocytes. Proceedings of the National Academy of Sciences of the United States of America. 2012;109(52):21319-24.

84. Lan X, Koutelou E, Schibler AC, Chen YC, Grant PA, Dent SY. Poly(Q) Expansions in ATXN7 Affect Solubility but Not Activity of the SAGA Deubiquitinating Module. Molecular and cellular biology. 2015;35(10):1777-87.

\section{Tables}




\begin{tabular}{|c|c|c|c|c|c|c|}
\hline \multirow[b]{2}{*}{ Gene symbol } & \multirow[b]{2}{*}{ Gene name } & \multirow{2}{*}{$\begin{array}{c}\text { SCA7 } \\
\log 2 F \mathrm{C} \\
\text { (SCA7w1) } \\
40 \mathrm{wk}\end{array}$} & \multicolumn{3}{|c|}{ SCA1 } & \multirow{2}{*}{ 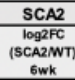 } \\
\hline & & & $\begin{array}{c}\begin{array}{c}\log 22 \mathrm{C} \\
\text { (SCA1wK) } \\
5 \mathrm{wwk}\end{array} \\
\end{array}$ & $\begin{array}{c}\log 22 \mathrm{C} \\
\text { (scA1w) } \\
12 \mathrm{wk} \\
\end{array}$ & $\begin{array}{c}\text { log2FC } \\
\text { (SCA1WT) } \\
28 \mathrm{wk} \\
\end{array}$ & \\
\hline$A b r^{r}$ & active BCR-related gene & -0.32 & -0.27 & -0.02 & -0.20 & -0.46 \\
\hline Ankrd33b & ankyrin repeat domain 33B & -0.52 & -0.49 & -1.28 & -1.58 & -0.73 \\
\hline Arhgap20 & Rho GTPase activating protein 20 & -0.73 & -0.47 & -0.79 & -1.22 & -0.70 \\
\hline Arthgap26 & Rho GTPase activating protein 26 & -0.62 & ns & -0.68 & -0.84 & -0.94 \\
\hline Arhgap31 & Rho GTPase activating protein 31 & -0.37 & ns & -0.64 & -1.12 & -0.70 \\
\hline Arhgap 32 & Rho GTPase activating protein 32 & -0.39 & ns & -0.49 & -1.03 & -0.69 \\
\hline Arhgap5 & Rho GTPase activating protein 5 & -0.49 & -0.26 & -1.22 & -1.05 & -0.56 \\
\hline Atp2a3 & ATPase, Ca++ transporting, ubiquitous & -0.38 & -0.79 & -1.50 & -1.84 & -1.26 \\
\hline B4gaint3 & beta-1,4-N-acetyl-galactosaminyi transferase 3 & -0.55 & ns & -4.15 & -0.56 & -1.77 \\
\hline Cacnb2' & calcium channel, voltage-dependent, beta 2 subunit & -0.42 & -0.51 & -0.92 & -1.05 & -0.49 \\
\hline Calb1 ${ }^{\circ \prime}$ & calbindin 1 & -0.47 & -0.61 & -1.17 & -1.42 & -0.87 \\
\hline Car8 ${ }^{\text {r }}$ & carbonic anhydrase 8 & -0.51 & -0.73 & -1.59 & -1.51 & -1.13 \\
\hline Casq2 & calsequestrin 2 & -0.42 & ns & -0.73 & -1.03 & -0.58 \\
\hline Clmn & calmin & -0.65 & -0.70 & -1.32 & -1.84 & -1.34 \\
\hline Corin & corin & -0.53 & -0.86 & -2.11 & -2.83 & -1.22 \\
\hline Creg1 & cellular repressor of E1A-stimulated genes 1 & -0.49 & -0.78 & -0.85 & -0.97 & -0.31 \\
\hline Cyth3 & cytohesin 3 & -0.37 & -0.42 & -0.79 & -0.65 & -0.56 \\
\hline Dagla * & diacylglycerol lipase, alpha & -0.45 & -0.90 & -1.12 & -1.44 & -1.58 \\
\hline Dap' & death-associated protein & -0.35 & -1.09 & -0.94 & -1.31 & -0.64 \\
\hline Dgkh' & diacylglycerol kinase, eta & -0.77 & -1.06 & -1.80 & -2.37 & -1.14 \\
\hline Dig2 & discs large MAGUK scaffold protein 2 & -0.43 & ns & -0.93 & -0.98 & -0.43 \\
\hline Dner & delta/notch-like EGF repeat containing & -0.36 & -0.56 & -1.13 & -1.08 & -1.20 \\
\hline$D o c 2 b$ & double C2, beta & -0.64 & -1.01 & -1.43 & -1.87 & -1.53 \\
\hline Et/4 & enhancer trap locus 4 & -0.50 & -0.35 & -0.51 & -0.72 & -0.82 \\
\hline Fam107b ' & family with sequence similarity 107 , member B & -1.03 & -1.08 & -1.92 & -1.65 & -1.87 \\
\hline Fam117a & family with sequence similarity 117 , member $A$ & -0.68 & ns & -0.82 & -0.60 & -1.18 \\
\hline Fam78b ' & family with sequence similarity 78 , member $B$ & -0.41 & ns & -0.45 & -0.85 & -0.60 \\
\hline Far2 & fatty acyl CoA reductase 2 & -0.54 & -0.58 & -1.36 & -1.22 & -0.70 \\
\hline Fgf7 & fibroblast growth factor 7 & -0.51 & -1.55 & -3.26 & -2.59 & -1.41 \\
\hline Gabbr1 & gamma-aminobutyric acid (GABA) B receptor, 1 & -0.37 & -0.57 & -0.69 & -0.76 & -0.53 \\
\hline Garn/3 & GTPase activating RANGAP domain-like 3 & -0.67 & -0.65 & -1.12 & -1.41 & -0.96 \\
\hline Gm5083 & predicted gene 5083 & -0.47 & -0.68 & -0.90 & -0.98 & -1.08 \\
\hline Gng13 & guanine nucleotide binding protein (G protein), gamma 13 & -0.74 & -1.30 & -1.92 & -1.97 & -1.54 \\
\hline Gpr63 & G protein-coupled receptor 63 & -0.90 & -1.07 & -1.93 & -2.58 & -2.17 \\
\hline Grid2" & glutamate receptor, ionotropic, delta 2 & -0.50 & -0.42 & -1.18 & -1.32 & -0.80 \\
\hline Grid2ip & glutamate receptor, ionotropic, delta 2 (Grid2) interacting protein 1 & -0.66 & -1.09 & -1.66 & -2.95 & -1.68 \\
\hline Htr1b & 5-hydroxytryptamine (serotonin) receptor $1 \mathrm{~B}$ & -0.45 & -0.52 & -1.36 & -1.21 & -1.16 \\
\hline Itemt' ${ }^{\prime}$ & isoprenylcysteine carboxyl methyltransferase & -0.54 & -0.63 & -1.41 & -1.56 & -0.88 \\
\hline Id $2^{\prime}$ & inhibitor of DNA binding 2 & -0.32 & -0.46 & -0.59 & -0.52 & -0.49 \\
\hline Inpp4a * & inositol polyphosphate-4-phosphatase, type I & -0.29 & -0.35 & -0.72 & -0.90 & -1.00 \\
\hline Inpp5a " & inositol polyphosphate-5-phosphatase A & -0.41 & -0.56 & -0.89 & -1.07 & -1.00 \\
\hline Atpri or & inositol 1,4,5-trisphosphate receptor 1 & -0.56 & ns & -1.43 & -2.00 & -1.00 \\
\hline Kena6' & potassium voltage-gated channel, shaker-related, subfamily, member 6 & -0.45 & -0.49 & -0.77 & -0.47 & -0.70 \\
\hline Kcnab1' & potassium voltage-gated channel, shaker-related subfamily, beta member 1 & -0.34 & -0.51 & -1.30 & -1.07 & -0.74 \\
\hline Kcnip1 & Kv channel-interacting protein 1 & -0.34 & -0.62 & -0.97 & -1.10 & -0.38 \\
\hline Kcnma1 * & potassium large conductance calcium-activated channel, subfamily M, alpha member 1 & -0.67 & -0.37 & -0.97 & -1.62 & -0.83 \\
\hline Kctd12 & potassium channel tetramerisation domain containing 12 & -0.95 & -0.87 & -1.96 & -1.44 & -1.23 \\
\hline Mtss1 ${ }^{\text {ar }}$ & metastasis suppressor 1 & -0.36 & -0.27 & -0.59 & -0.88 & -0.93 \\
\hline Nexn & nexilin & -0.36 & ns & -0.67 & -0.81 & -0.62 \\
\hline Opn3 & opsin 3 & -1.03 & -1.05 & -1.74 & -0.76 & -1.94 \\
\hline Pcp4' ${ }^{\prime}$ & Purkinje cell protein 4 & -0.57 & -0.85 & -1.14 & -1.30 & -0.81 \\
\hline Pde5a & phosphodiesterase 5 A cGMP-specific & -0.70 & -0.45 & -1.50 & -1.83 & -0.89 \\
\hline Pde9a & phosphodiesterase 9A & -0.46 & -0.37 & -0.12 & -0.47 & -0.47 \\
\hline Plcb3' & phospholipase C, beta 3 & -0.72 & -0.91 & -0.92 & -1.10 & -0.98 \\
\hline Plekhd1 & pleckstrin homology domain containing, family $\mathrm{D}$ (with colled-coll domains) member 1 & -0.34 & -0.39 & -0.74 & -0.78 & -0.61 \\
\hline Pogk & pogo transposable element with KRAB domain & -0.45 & -0.32 & -0.75 & -0.67 & -0.54 \\
\hline Ppp1r16b & protein phosphatase 1 , regulatory subunit $16 \mathrm{~B}$ & -0.72 & -0.74 & -1.31 & -1.44 & -1.25 \\
\hline Ppp1r17 & protein phosphatase 1 , regulatory subunit 17 & -0.34 & -0.45 & -1.08 & -0.93 & -0.65 \\
\hline Ppp4r4 & protein phosphatase 4 , regulatory subunit 4 & -0.49 & -0.46 & -0.98 & -1.26 & -0.70 \\
\hline Prkg1 & protein kinase, cGMP-dependent, type I & -0.80 & -0.48 & -1.05 & -1.20 & -0.90 \\
\hline Prmt8 & protein arginine $\mathrm{N}$-methyltransferase 8 & -0.52 & -0.35 & -0.49 & -0.50 & -0.70 \\
\hline Psd2 & pleckstrin and Sec7 domain containing 2 & -0.31 & -0.38 & -0.57 & -0.38 & -0.49 \\
\hline Ptprr ${ }^{2}$ r & protein tyrosine phosphatase, receptor type, $R$ & -0.36 & -0.36 & -1.14 & -0.83 & -0.50 \\
\hline Rdh11 & retinol dehydrogenase 11 & -0.46 & -0.40 & -0.90 & -0.60 & -0.45 \\
\hline Rgs7bp & regulator of G-protein signalling 7 binding protein & -0.32 & ns & -0.87 & -0.64 & -0.33 \\
\hline Rgse & regulator of G-protein signaling 8 & -0.91 & -0.97 & -2.06 & -2.13 & -1.50 \\
\hline Rnf19b & ring finger protein 198 & -0.45 & -0.49 & -0.70 & -0.68 & -0.90 \\
\hline Rora ar & RAR-related orphan receptor alpha & -0.39 & ns & -0.87 & -1.25 & -0.63 \\
\hline Rtel1 & regulator of telomere elongation helicase 1 & -0.32 & -0.55 & -0.79 & -0.71 & -0.41 \\
\hline Samd8 & sterile alpha motif domain containing 8 & -0.33 & -0.43 & -0.90 & -0.92 & -0.60 \\
\hline Slc9a3 ${ }^{r}$ & solute carrier family 9 (sodium/mydrogen exchanger), member 3 & -0.60 & -0.82 & -1.66 & -2.00 & -1.22 \\
\hline SrI & sarcalumenin & -0.77 & -0.91 & -1.24 & -1.04 & -0.78 \\
\hline Stac & src homology three (SH3) and cysteine rich domain & -0.66 & -0.92 & -1.26 & -1.83 & -1.22 \\
\hline Stk17b & serine/threonine kinase 17b (apoptosis-inducing) & -0.71 & -0.84 & -1.86 & -1.74 & -1.02 \\
\hline Strip2 & striatin interacting protein 2 & -0.49 & -0.45 & -1.37 & -1.24 & -0.73 \\
\hline Strn3 & striatin, calmodulin binding protein 3 & -0.34 & -0.42 & -0.75 & -0.87 & -0.37 \\
\hline Tmem64 & transmembrane protein 64 & -0.33 & -0.27 & -0.85 & -0.58 & -0.37 \\
\hline Trpc3 ${ }^{\circ}$ & Transient Receptor Potential Cation Channel Subfamily C Member 3 & -0.50 & -0.65 & -1.57 & -1.58 & -1.26 \\
\hline 2410124H12Rik & RKEN cDNA 2410124H12 gene & -0.88 & -1.75 & -3.11 & -3.67 & -2.08 \\
\hline 3632451006 Rik & - & -0.40 & ns & -0.84 & -0.53 & -0.25 \\
\hline
\end{tabular}

ns: not significant, ----- no name available, a: genes associated with genetic ataxias, r: RORa genetic targets

Figures 

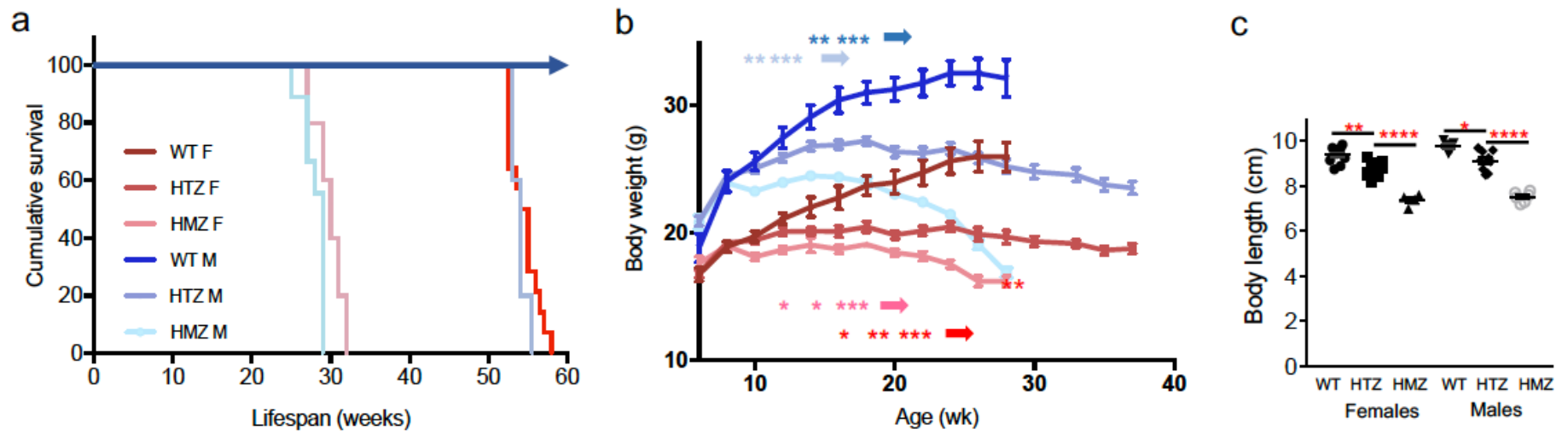

\section{Figure 1}

Lifespan and body size of SCA7 mice. a Kaplan-Meier survival curves indicate that SCA7140Q/5Q heterozygous (HTZ) and SCA7140Q/140Q homozygous (HMZ) males (M) and females (F) have shorter lifespan than wild type (WT) littermates; $n=$ WT F: 11; WT M: 5; HTZ F: 9; HTZ M: 4; HMZ F: 6; HMZ M: 5. $b$ Gain in body weight of females and males of SCA7 HTZ and SCA7 HMZ mice differs from WT littermates; n= WT F: 11; WT M: 5; HTZ F: 8; HTZ M:9; HMZ F:6; HMZ M:9). c Body length of 38-week old females and males of WT, SCA7 HTZ and SCA7 HMZ mice. Data are expressed as mean \pm SEM. b, Twoway ANOVA with repeated measurement followed by post-hoc Bonferroni test. c, One-way ANOVA followed by post-hoc Bonferroni test. ${ }^{*} p<0.05 ;{ }^{* \star} p<0.01 ;{ }^{* \star *} p<0.001 ;{ }^{* \star * *} p<0.0001$. 

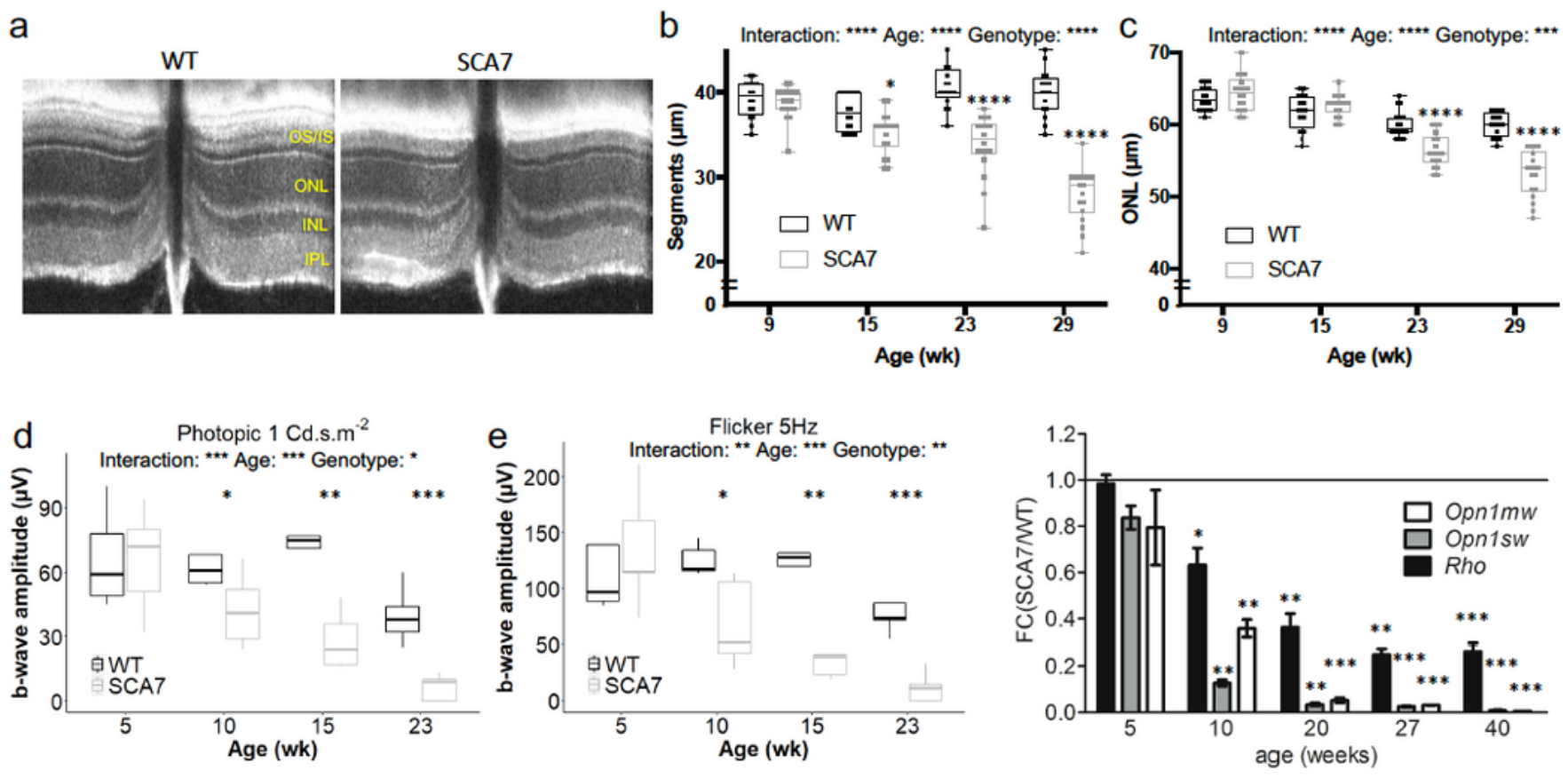

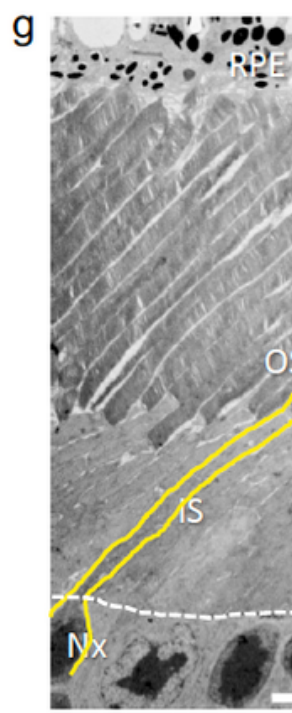

WT

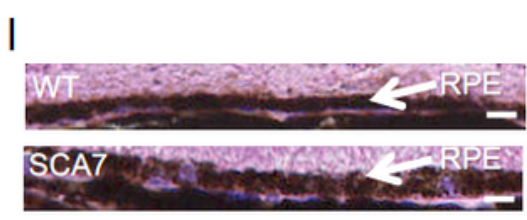

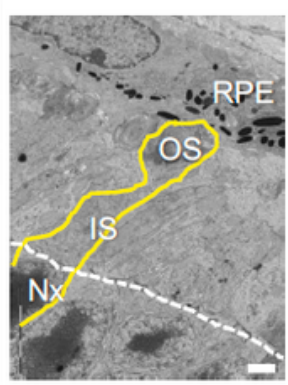

SCA7 Hmz

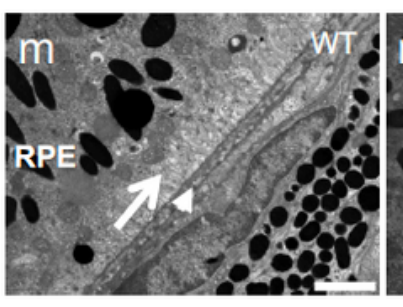

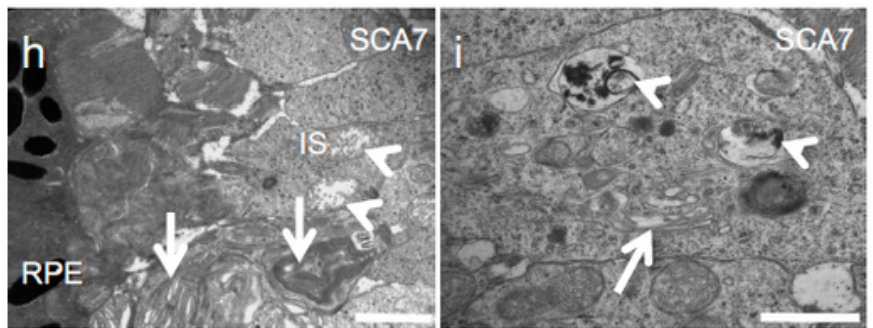
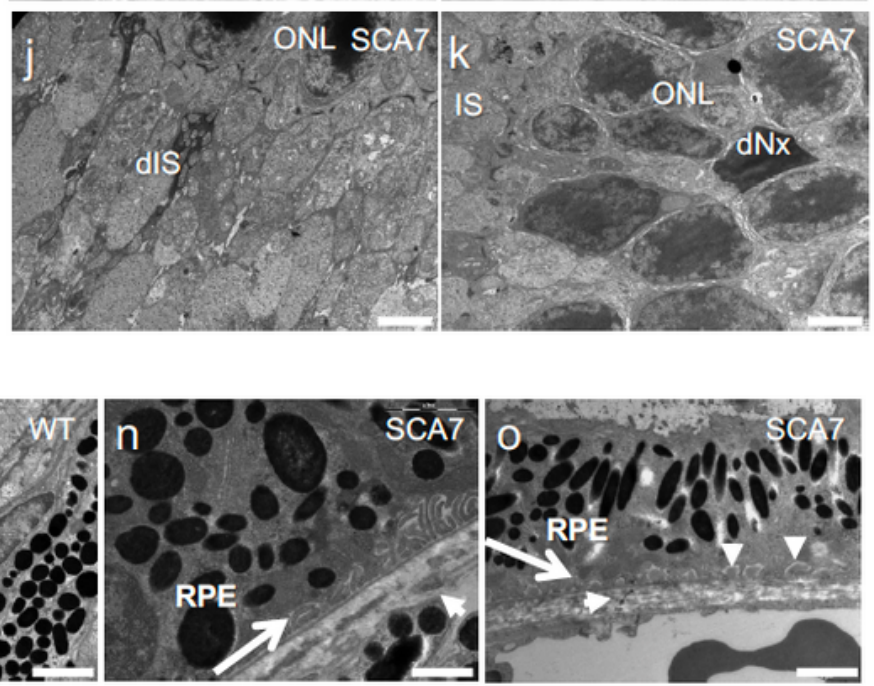

\section{Figure 2}

Photoreceptor and retinal pigmented epithelium degeneration in SCA7 mice. a Representative optical coherence tomography (OCT) B-scan showing reduced thickness of the segment layers of SCA7140Q/5Q mice compared to wild type (WT) at 29 weeks (wk). Outer segment (OS) and inner segment (IS), outer nuclear layer (ONL) inner nuclear layer (INL), inner plexiform layer (IPL). b-c Longitudinal OCT measurements show progressive thinning of photoreceptor segment layers (b) and ONL (c) in 
SCA7140Q/5Q mice relative to WT littermates; $n=$ both eyes of 8 WT and 9 SCA7 mice. d-e Longitudinal cone-derived b-wave amplitudes of photopic response to $1 \mathrm{Cd}$.s.m-2 flashes (d) and $5 \mathrm{~Hz}$ flicker responses to 3 Cd.s.m-2 flashes (e) show progressive reduction of cone function in SCA7140Q/5Q retina relative to WT retina; $n=$ single eyes of 5 WT and 5 SCA7 mice. Background illumination was $25 \mathrm{Cd} . \mathrm{m}-2$. $f$ Quantitative RT-PCR analysis shows early and progressive downregulation of Opn $1 \mathrm{mw}$ and Opn $1 \mathrm{sw}$ cone genes and Rhodopsin (Rho) rod gene in SCA7140Q/5Q retina, as shown by normalization to WT gene expression levels; $n=3-6 /$ genotype. $g$ Electron micrographs showing the progressive disappearance of OS and the shortening of IS of 44 wk-old SCA7 heterozygotes (Htz) and 20 wk-old SCA7 homozygotes (Hmz), compared to WT mice. Retinal pigmented epithelium (RPE), photoreceptor nuclei (Nx). Scale bar $=10 \mu \mathrm{m}$. h-k Electron micrographs of 20 wk-old SCA7140Q/140Q retina. h, the remnant OS have lost their parallel organization (arrows) and the IS contain swollen and disrupted mitochondria (arrowheads). i, The IS shows dilated and vesiculated endoplasmic reticulum (arrow) and accumulation of vesicular membranes (arrowhead). j, Dark degenerating IS (dIS) with numerous abnormal mitochondria. k, Dark photoreceptor nuclei $(\mathrm{dNx})$. Scale bar: $2 \mu \mathrm{m}(\mathrm{H}, \mathrm{I})$ and $5 \mu \mathrm{m}(\mathrm{J}, \mathrm{K})$. I Histological sections comparing the thickness of WT and SCA7 RPE at $20 \mathrm{wk}$. Scale bar $=15 \mu \mathrm{m}$. m-o, Electron micrographs comparing the basal infolding membrane of RPE and Bruch's membrane of WT and SCA7 mice. In WT (m), the basal infolding membrane of RPE (arrow) shows a typical lamellar organization with translucid lumen, and a regular Bruch's membrane (arrowhead). In contrast in SCA7 retina, the infolding membrane (long arrow) is either opaque (n) or completely absent (o), while the Bruch's membrane (short arrow) is enlarged and disorganized $(n, 0)$, and occasionally interrupted $(n)$. In addition, homogenous deposits are found between the basement membrane and plasma membrane of RPE (vertical arrowheads in 0). Scale bar $=$ $2 \mu \mathrm{m}$. Data are expressed as mean \pm SEM and analyzed using Student's t-test in f; box-and-whisker plot and two-way ANOVA with repeated measurement followed by post-hoc Bonferroni test in b-e.: ${ }^{*} p<0.05$; $\star \star p<0.01 ; * \star * p<0.001$ 

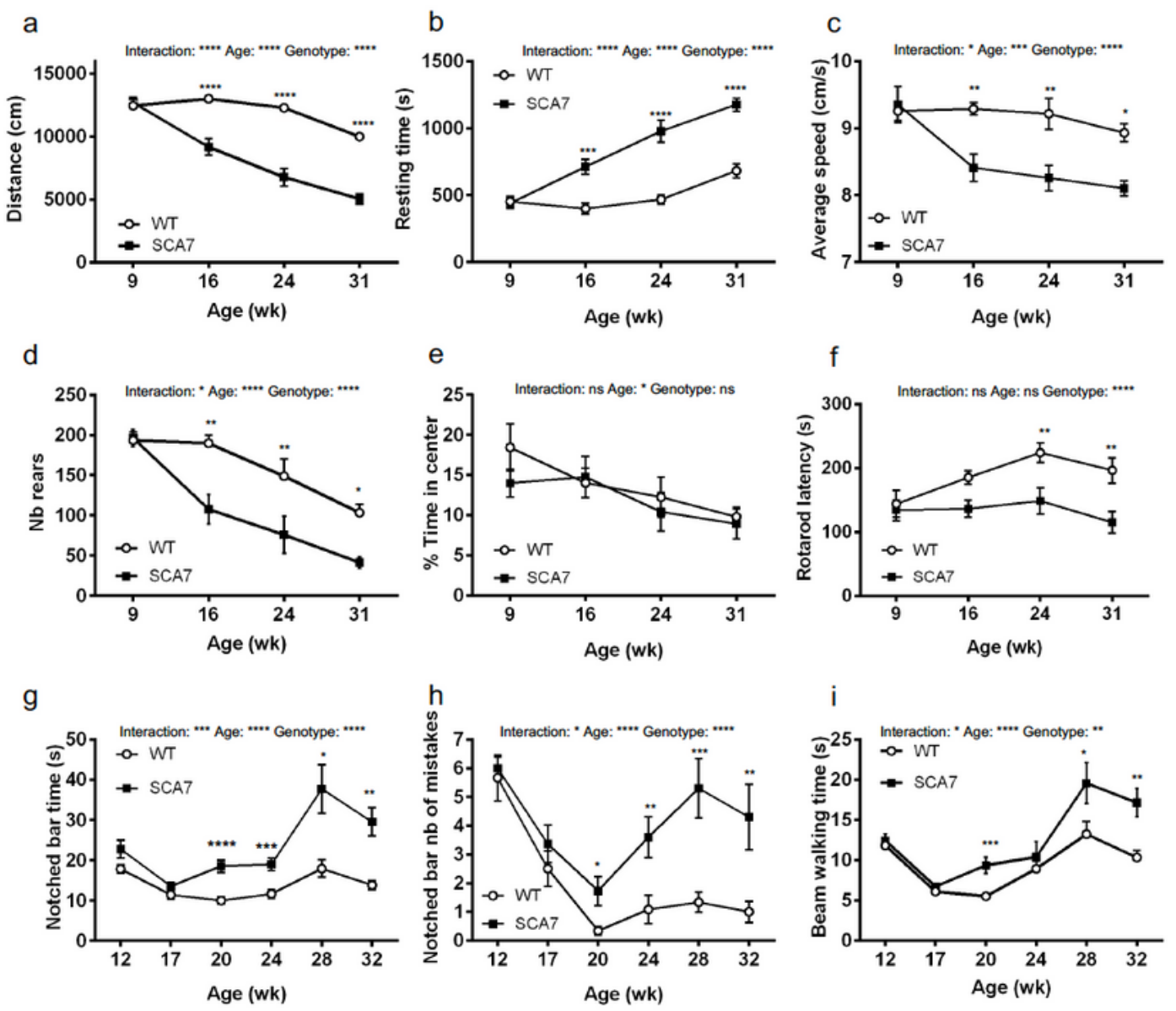

$$
\text { j }
$$

$$
\text { k }
$$
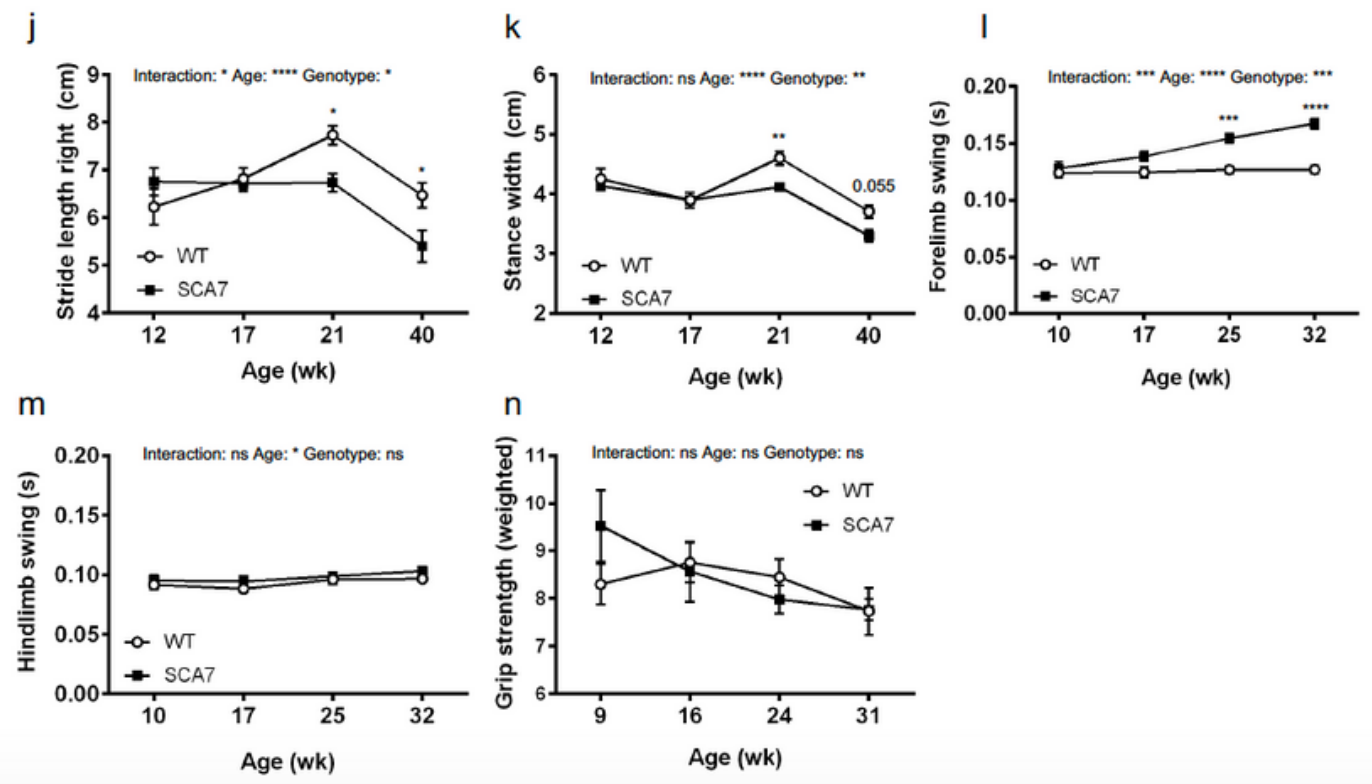

\section{Figure 3}

Behavioral and motor deficits of SCA7 mice. a-e Open field analyses show that SCA7140Q/5Q males ( $\mathrm{n}=$ 6) progressively become hypoactive relative to wild type (WT) male littermates ( $n=6)$, according to the distance travelled (a), the resting time (b), the average speed (c) and the number ( $\mathrm{Nb}$ ) of rears (d). However, SCA7 mice show no difference with WT mice as for the time spent in the arena center (e). Centimeter (cm), second (s), week (wk). f Accelerating rotarod (mean latency to fall) shows progressive 
motor deficit of SCA7140Q/5Q mice $(n=6)$ compared to WT littermates $(n=6)$. g-h Notched bar (time to cross the bar ( $\mathrm{g}$ ) and number of mistakes $(\mathrm{h}))$ indicates that SCA7140Q/5Q mice $(\mathrm{n}=10)$ progressively have altered motor coordination and balance compared to WT littermates $(n=12)$. i Beam walking also shows that motor performances of SCA7140Q/5Q $(n=6)$ mice are affected compared to WT littermates $(n=6)$. j-k Spatial gait analysis using footprint patterns indicates that SCA7140Q/5Q mice $(n=9-11)$ progressively have shorter stride length of the four limbs (right forelimb illustrated in $\mathrm{j}$ ) and shorter stance width (forelimbs illustrated in k) compared to WT littermates ( $n=9-12)$. I-m Temporal gait analysis using Catwalk shows that swing times of forelimbs (average of right and left forelimbs in I), but not hindlimbs (average of right and left hindlimbs in $m$ ) of SCA7140Q/5Q mice $(n=15)$ progressively become slower than those of WT littermates ( $n=10)$. $n$ Grip strength performance of SCA7140Q/5Q mice $(n=6)$ normalized by mouse body weight is similar to WT mice $(n=6)$. Data are expressed as mean \pm SEM. Twoway ANOVA with repeated measurement (a-i, l-n) or ordinary two-way ANOVA (j, k), followed by post-hoc Bonferroni test: ${ }^{*} p<0.05 ;{ }^{* \star} p<0.01 ;{ }^{* \star *} p<0.001 ;{ }^{* \star \star *} p<0.0001$; ns $=$ not significant. 

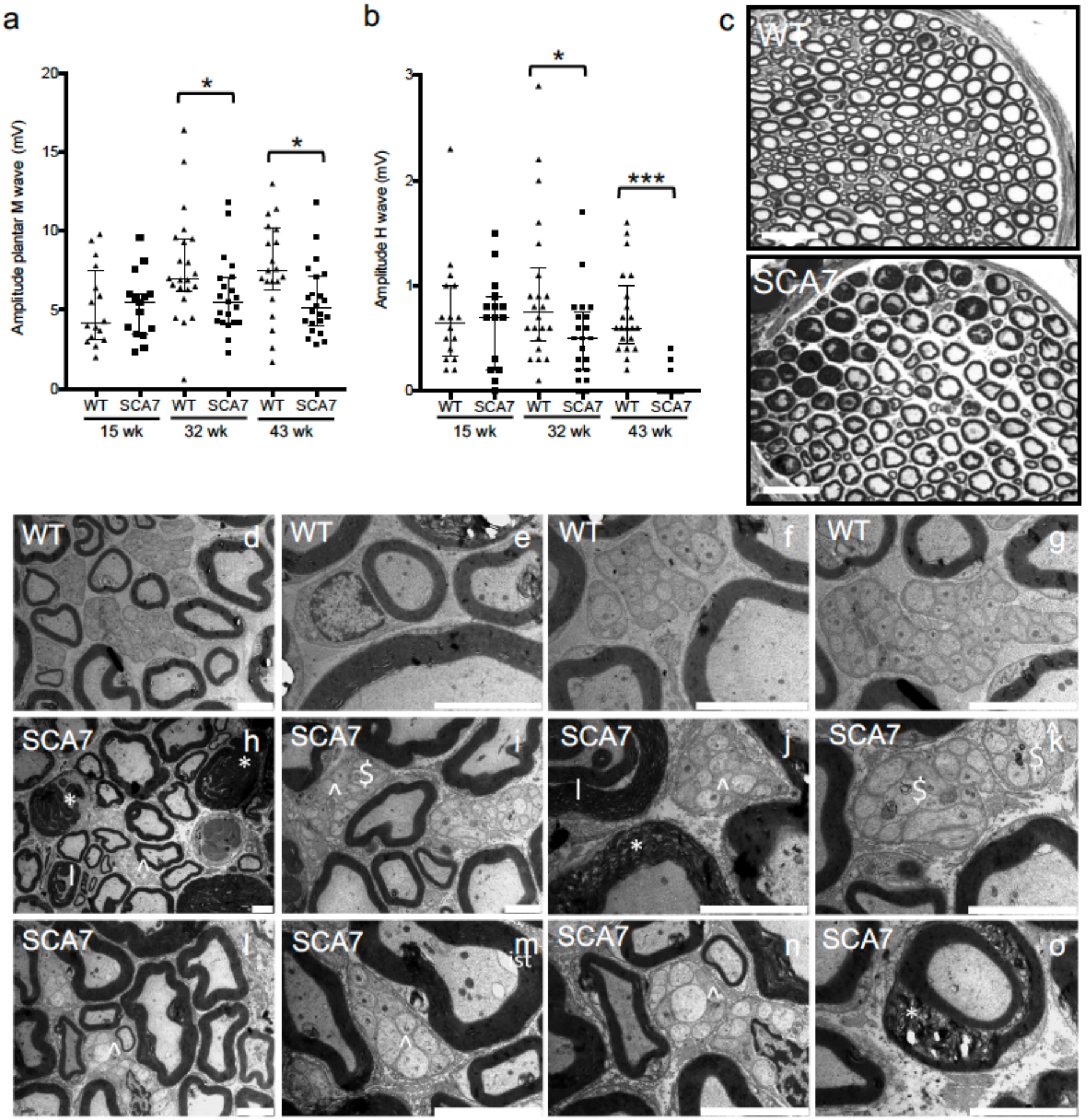

Figure 4

Peripheral nerve alterations in SCA7 mice. $a$, b Electromyograph activities of the sciatic nerve. Amplitude of motor M-wave (a) and sensory H-wave (b) in SCA7140Q/5Q mice relative to wild type (WT) littermates (15 weeks (wk): $n=16$ WT and 15 SCA7; 32 wk: $n=22$ WT and 21 SCA7; 43 wk: $n=22$ WT and 21 SCA7). Scatter plot showing median with interquartile range. Mann-Whitney test: ${ }^{\star} p<0.05$, ${ }^{\star \star \star} p<0.001$. c Comparison of semithin sections of sciatic nerve of SCA7140Q/5Q and WT mice. The mutant shows 
irregular and degenerated myelinated fibers and loss of small fibers. Scale bars, $50 \mu \mathrm{m}$ d-o Electron microscopy analyses of the sciatic nerve. Compared to WT littermates (d-g), sciatic nerve section of SCA7140Q/5Q mice (h-o) at 43 wk shows severe abnormalities in both myelinated and non-myelinated fibers including myelin degeneration (* in $h, j, o$ ), infolding like structure (I in $h, j)$, abnormal Remak bundle ( ${ }^{\wedge}$ in $\mathrm{h}-\mathrm{j}, \mathrm{I}-\mathrm{n}$ ), autophagy ( $\$$ in $\mathrm{i}, \mathrm{k}$ ), inner swelling tongue (ist in $\mathrm{m}$ ). Scales bar: $\mathrm{D}, \mathrm{M}$ and $\mathrm{N}=5 \mu \mathrm{m}$; E-G, I-L and $\mathrm{O}=2 \mu \mathrm{m} ; \mathrm{H}=10 \mu \mathrm{m}$.
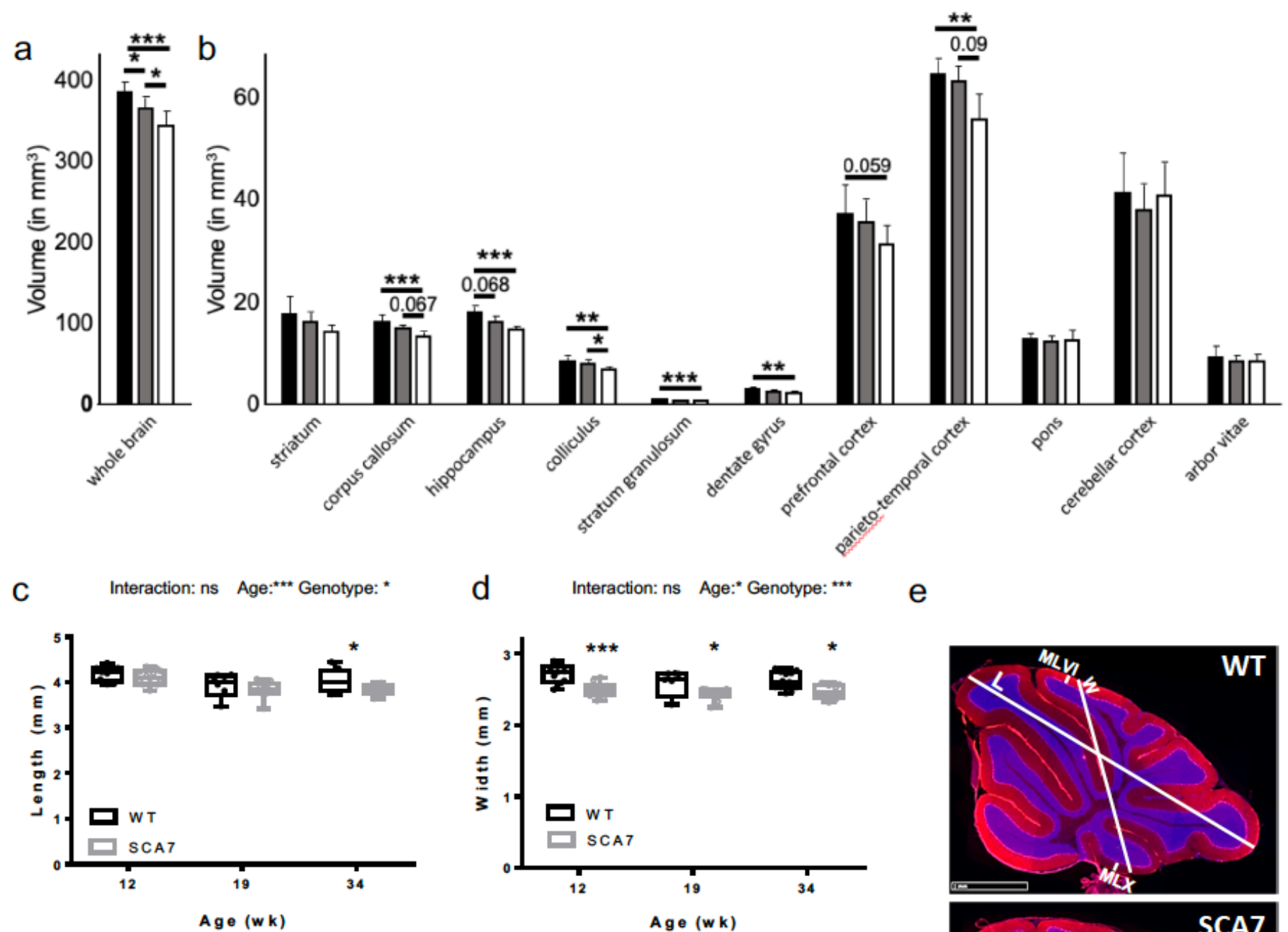

f Interaction: ns Age:* Genotype: *
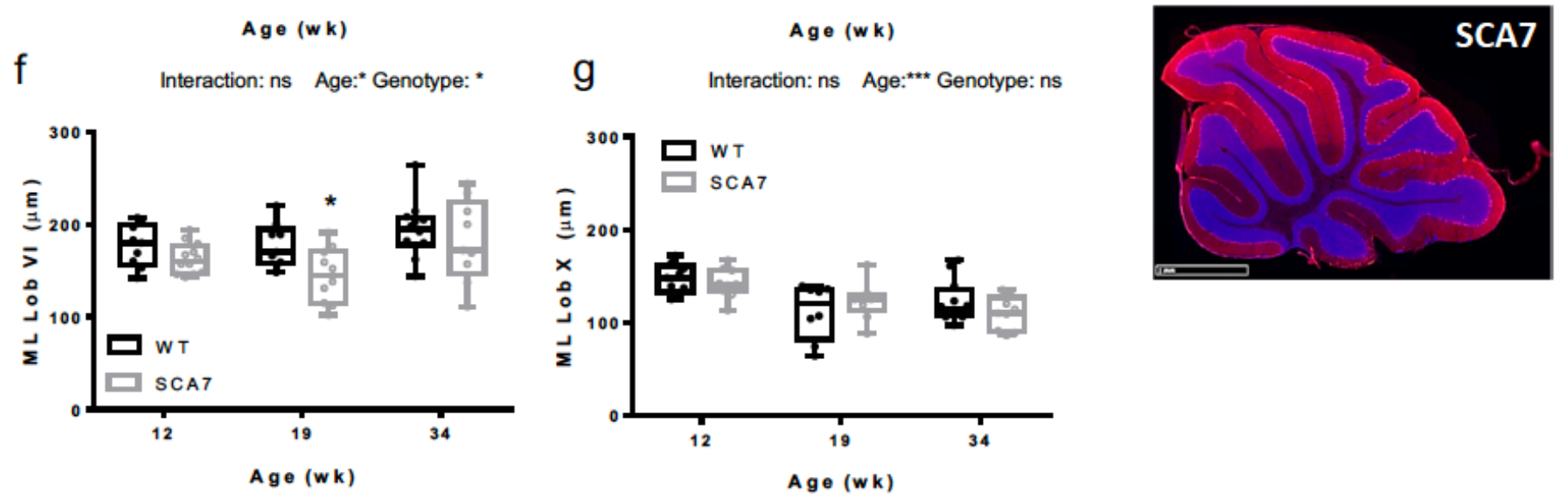

Figure 5 
Brain region atrophy of SCA7 mice. a, b Ex vivo MRI measurement of whole brain volume (a) and individual brain structures (b) showing differences between wild type (WT), SCA7140Q/5Q and SCA7140Q/140Q mice at 24 weeks (black, grey and white bars, respectively); $n=8$ WT, 9 SCA7140Q/5Q, 6 SCA7140Q/140Q mice. Cubic millimeter $=\mathrm{mm} 3 . \mathrm{c}, \mathrm{d}$ Measurement of vermis length $(\mathrm{c})$ of cerebellar sections (axis from lobule $V$ to IX in e) shows shortening at late disease stage (34 weeks (wk)) in SCA7140Q/5Q relative to WT mice, while the width (d) (axis from lobule VI to $X$ in e) has reduced size from 12 weeks onward in SCA7140Q/5Q mice; $n=8-12$ tissue sections/age-genotype. Millimeter = mm. e Representative cerebellar vermis section of WT and SCA7 mice immunolabeled with CALB1 antibody (red) and DAPI (blue). $\mathrm{f}, \mathrm{g}$ Measurement of the thickness of molecular layer (ML) of lobules (Lob) VI and $X$ of cerebellar sections shows no steady difference between SCA7140Q/5Q and WT mice; 9-12 sections $/$ mouse Micrometer $=\mu \mathrm{m}$. Data are expressed as mean \pm SEM. Statistical analysis was performed by one-way ANOVA with post-hoc Bonferroni test $(a, b)$ and ordinary two-way ANOVA with post-hoc Bonferroni test $(c, d, f, g) .{ }^{*} p<0.05, * \star p<0.01, * \star \star p<0.001$. ns $=$ not significant. 


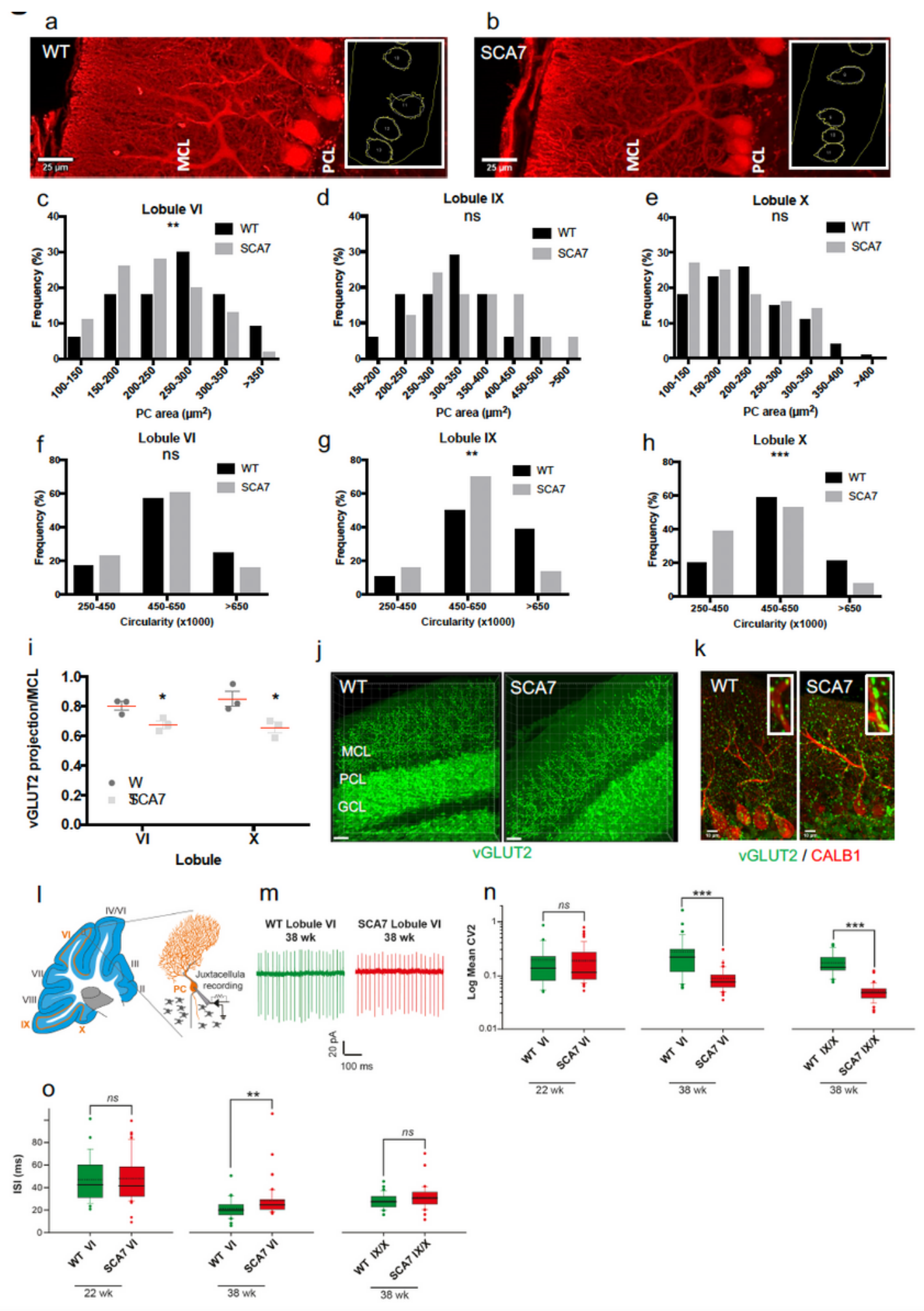

Figure 6

Alteration of morphology, synaptic contact and intrinsic activity of PC in SCA7 mice. a, b CALB 1 immunostaining (red) of Purkinje cells (PC) in lobule VI of the vermis in wild type (WT) and SCA7 mice (left). The staining is used to evaluate the soma size and cell circularity (right). Molecular cell layer (MCL); PC layer (PCL). Scale bar $=20 \mu \mathrm{m}$. c-h Frequency of PC soma area (c-e) and frequency of PC circularity index (f-h) as measured in lobule VI, IX and X of the vermis of 34-week-old WT (n=3) and SCA7140Q/5Q 
mice (n=3). 33-51 PC/lobule/mouse were measured. SCA7140Q/5Q mice show different distribution of PC area in lobule VI and different distribution of PC circularity in lobule IX and X, relative to WT mice. Square micrometer $(\mu \mathrm{m} 2)$. i, $j$ Width measurement of vGLUT2-labeled area in the molecular cell layer (MCL) of lobule VI and X of the vermis shows reduced width size in SCA7140Q/5Q $(n=3)$ compared to WT mice $(n=3)$. Data are expressed as mean \pm SEM. Granular cell layer $(G C L)$. Scale bar $=30 \mu m$. k Coimmunostaining of vGLUT2 and CALB1 showing aggregation of vGLUT2 contact points located along large dendritic arborization of PC in SCA7 cerebellum. Scale bar $=10 \mu \mathrm{m}$. I Left: Schematic of a sagittal cerebellar section. Cerebellar lobules are numbered from I to X. Orange lines indicate the locations of PC intrinsic activity recording sites. Right: schematic of juxtacellular recording of PC pacemaking activity. $\mathrm{m}$ Representative traces of spontaneous firing in PCs located in lobule VI from WT or SCA7140Q/5Q mice. Week (wk). n Box-plots showing the values of CV2 of adjacent inter-spike interval (ISI) in lobule VI and IX/X in WT and SCA7 mice. Mean and median values are indicated as dashed and solid lines, respectively (22 wk old WT ( $n=27)$ and SCA7 $(n=65) ; 38$ wk old WT $(n=33$ in lobule VI and 34 in IX/X) and SCA7 $(n=$ 41 in lobule VI and 36 in IX/X). o Same representation as in N for ISI values. Statistical analysis was performed using Chi-square (c-h), Student t-test (i) and Mann Whitney rank-sum test $(n, 0) .{ }^{*} p<0.05$; ${ }^{\star \star} p<0.01 ;{ }^{* \star *} p<0.001 ; n s=$ not significant 
a
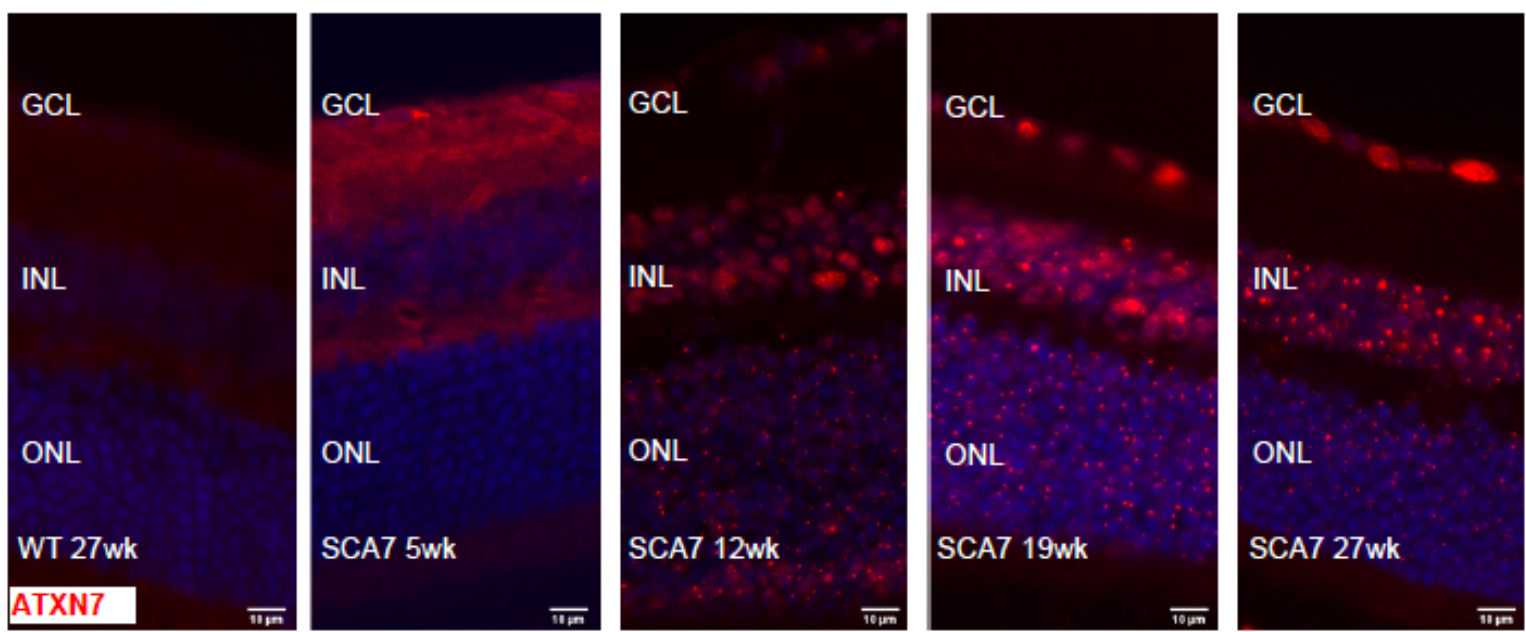

b PCL GCL MCL PCL GCL MCL PCL GCL MCL PCL GCL MCL PCL GCL MCL
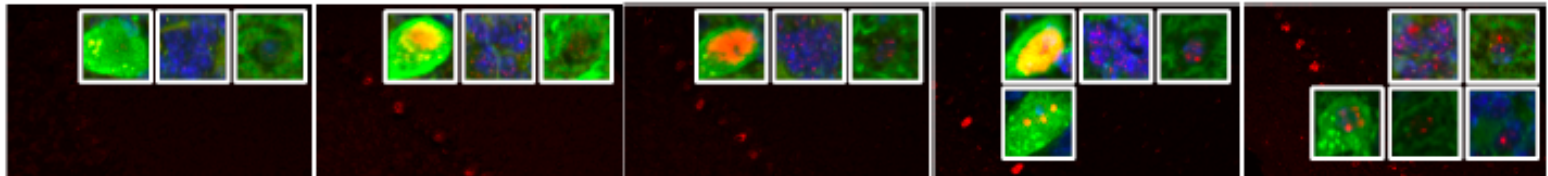

ATXN7

CALB1

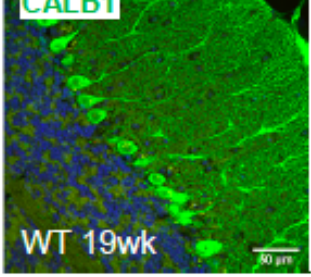

C

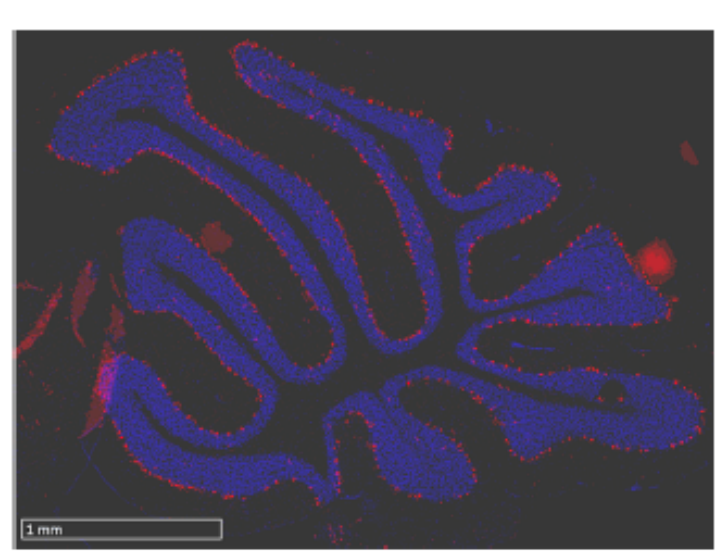

d

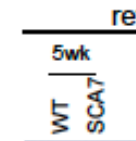

SCA7 27 wk
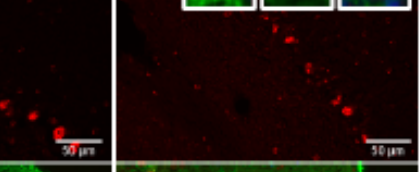

tina
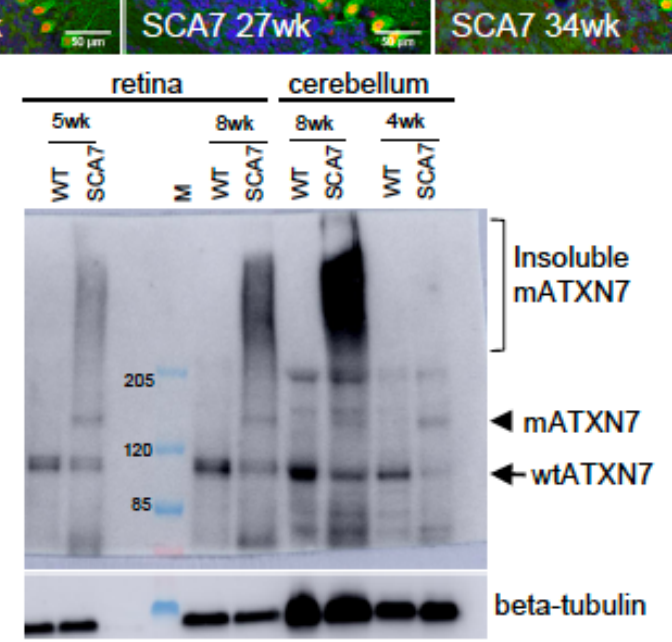

\section{Figure 7}

Accumulation of mutant ATXN7 in SCA7 mouse retina and cerebellum. a Immunostaining of ATXN7 (red) on retinal sections of wild type (WT) and SCA7140Q/5Q mice at different ages, showing the progressive accumulation of mutant ATXN7 (mATXN7) aggregates in retinal cells. DAPI-stained nuclei (blue). ONL, outer nuclear layer; INL, inner nuclear layer; GCL, ganglion cell layer. Week (wk). Scale bar = 10 $\mu \mathrm{m}$. b Coimmunostaining of ATXN7 (red) and CALB1 (green) of cerebellar sections, showing the progressive 
accumulation of mATXN7 aggregates in different cerebellar cells. DAPI-stained nuclei (blue). Purkinje cell layer (PCL), granular cell layer (GCL), molecular cell layer (MCL). Scale bar $=50 \mu \mathrm{m} . \mathrm{c} \mathrm{Immunostaining} \mathrm{of}$ ATXN7 (red) showing the global distribution of mATXN7 aggregates in the PC layer of SCA7 cerebellum. DAPI-stained nuclei (blue). Scale bar $=1 \mathrm{~mm}$. $\mathrm{d}$ Western blot analysis of whole protein extract of retina and cerebellum of WT and SCA7140Q/5Q mice at different ages, probed with ATXN7 and beta-tubulin antibodies. Arrows and arrowhead indicate the WT and mutant forms of ATXN7, respectively. The bracket indicates the insoluble high molecular weight mATXN7 material in SCA7 samples. Molecular weight (M) in kilodalton, week (wk). 
a
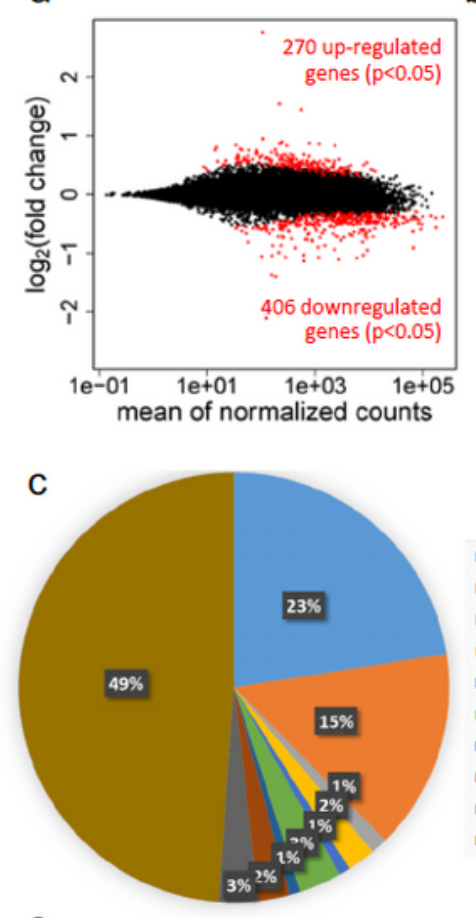

e

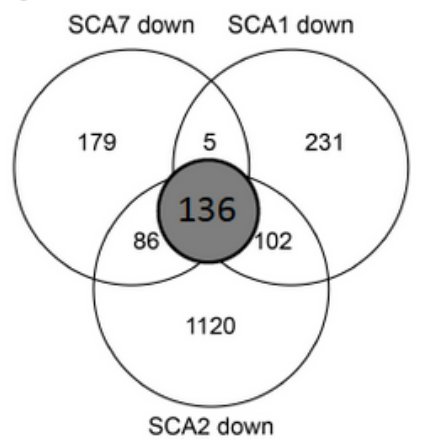

f

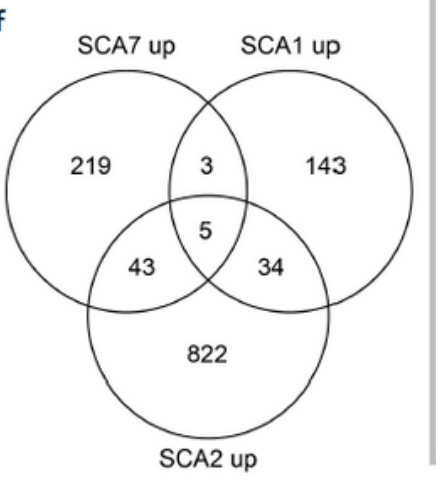

b
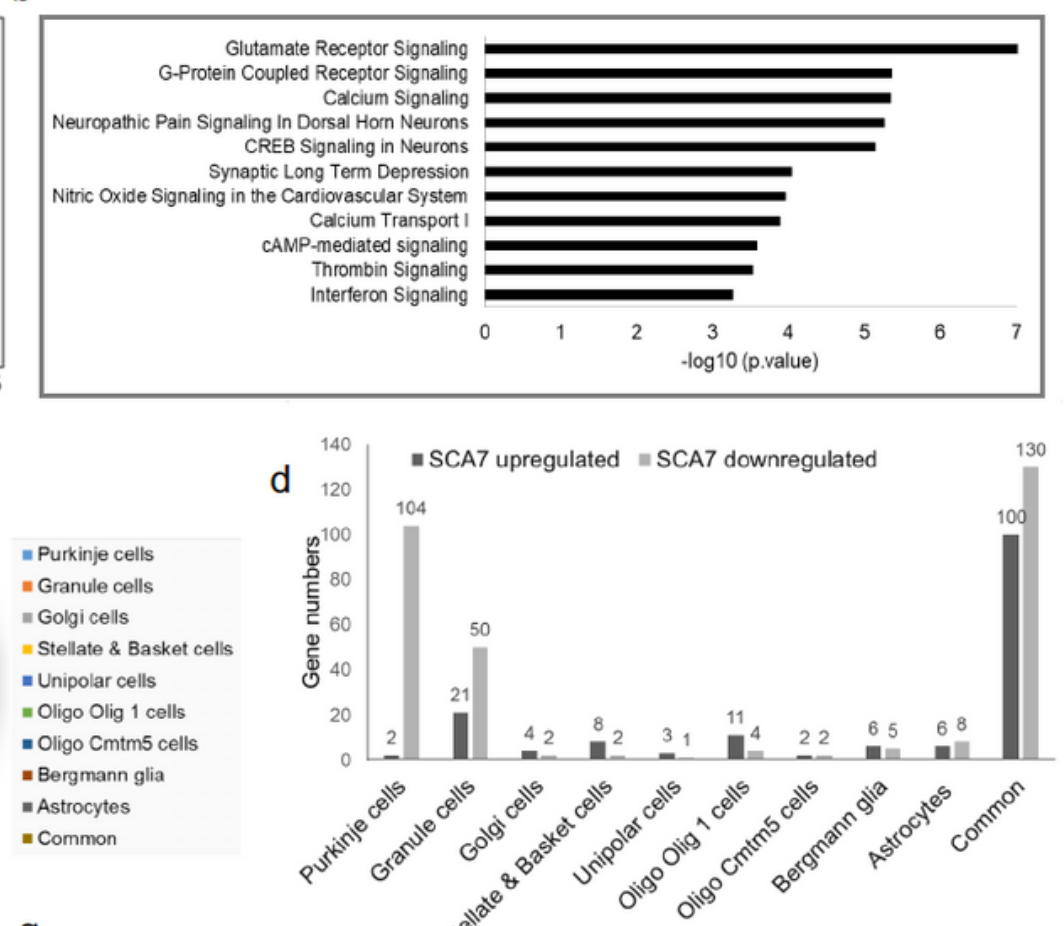

g

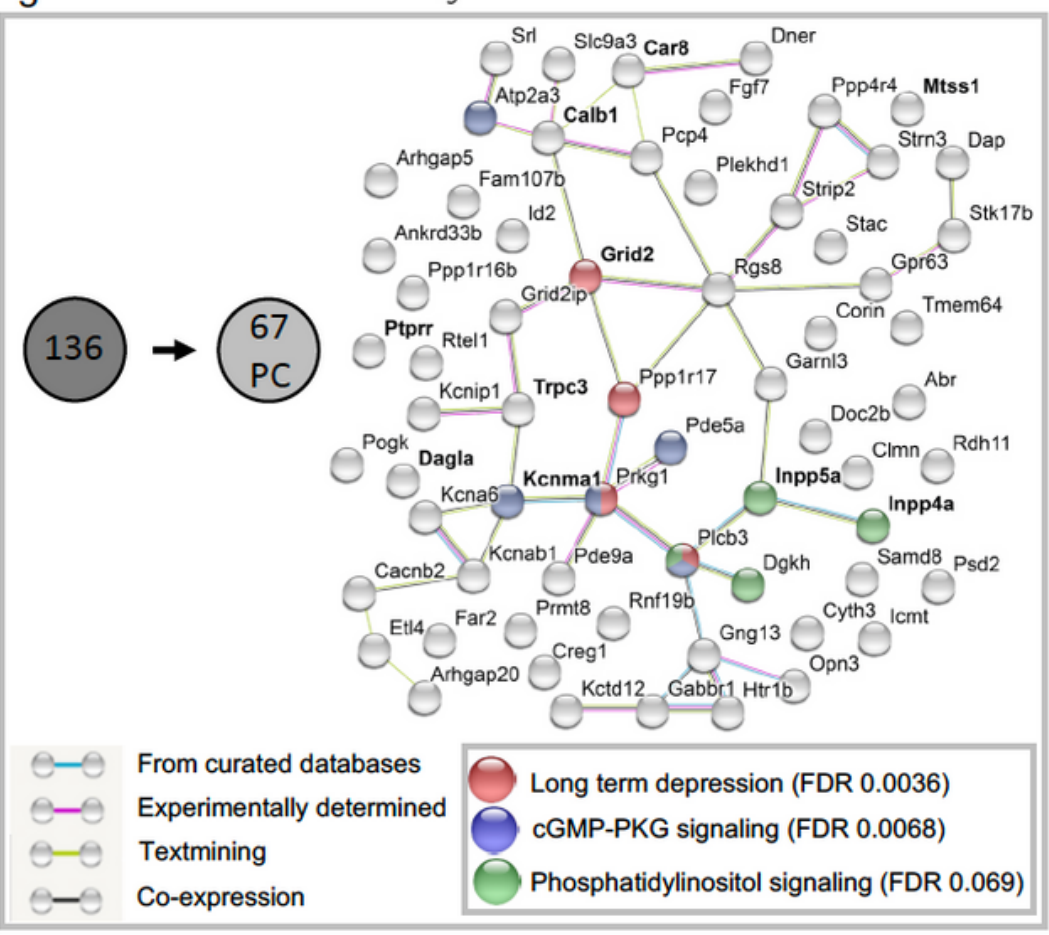

\section{Figure 8}

Gene deregulation, cell type identity and network analysis. a MA plot of transcriptomic dataset between SCA7140Q/5Q and wild type (WT) mice cerebellum at 40 weeks of age. Red points represent 676 genes deregulated in SCA7140Q/5Q ( $<<0.05)$. b Functional annotations of the deregulated genes performed using Ingenuity Pathway Analysis. The most significantly affected pathways in SCA7140Q/5Q are indicated. c Cerebellar cell type distribution of the genes deregulated in SCA7140Q/5Q mice based on 
curated dataset of Doyle et al. (2008) (37). d Cell type classification of up- and downregulated genes indicates Purkinje cells as the predominant cell type affected by gene downregulation in SCA7 cerebellum. Common means genes deregulated in more than one cell type. e, $\mathrm{f}$ Venn diagrams showing overlap between genes downregulated (e) and upregulated (f) in SCA1 (5 week-old) (47), SCA2 (6 weekold) (46) and SCA7140Q/5Q cerebellum. g Genetic network showing functional partnerships and interactions between proteins encoded by the 67 PC-enriched genes downregulated in SCA1, SCA2 and SCA7 mouse models, as determined using the STRING software. In bold are known ataxia genes. The bottom right panel indicates functional annotations of the 67 PC-enriched genes downregulated in SCA1, SCA2 and SCA7 mouse models, using KEGG pathway analysis. False discovery rate (FDR).
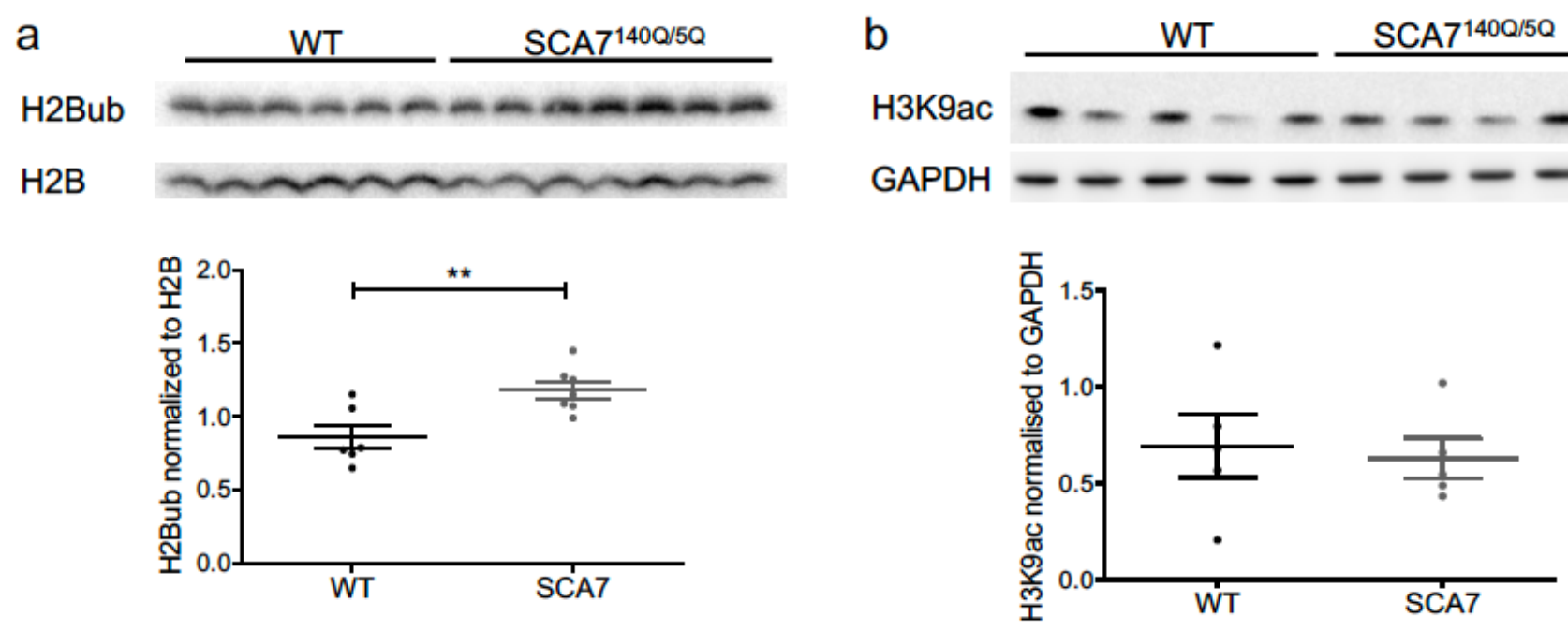

\section{Figure 9}

Alteration of SAGA-dependent histone marks in SCA7140Q/5Q cerebellum. Western blot analyses showing that (a) the bulk level of monoubiquitinated histone $\mathrm{H} 2 \mathrm{~B}$ ( $\mathrm{H} 2 \mathrm{Bub})$ is significantly increased in the cerebellum of SCA7140Q/5Q mice compared to wild type (WT), while (b) the bulk level of acetylated lysine 9 of histone H3 (H3K9ac) is comparable in SCA7140Q/5Q and WT mice. Immunodetected signals were quantified in Fiji and normalized using the level of H2B (a) and GAPDH (b). Data represent mean \pm SEM. Student's t-test, $* \star p<0.01$. 


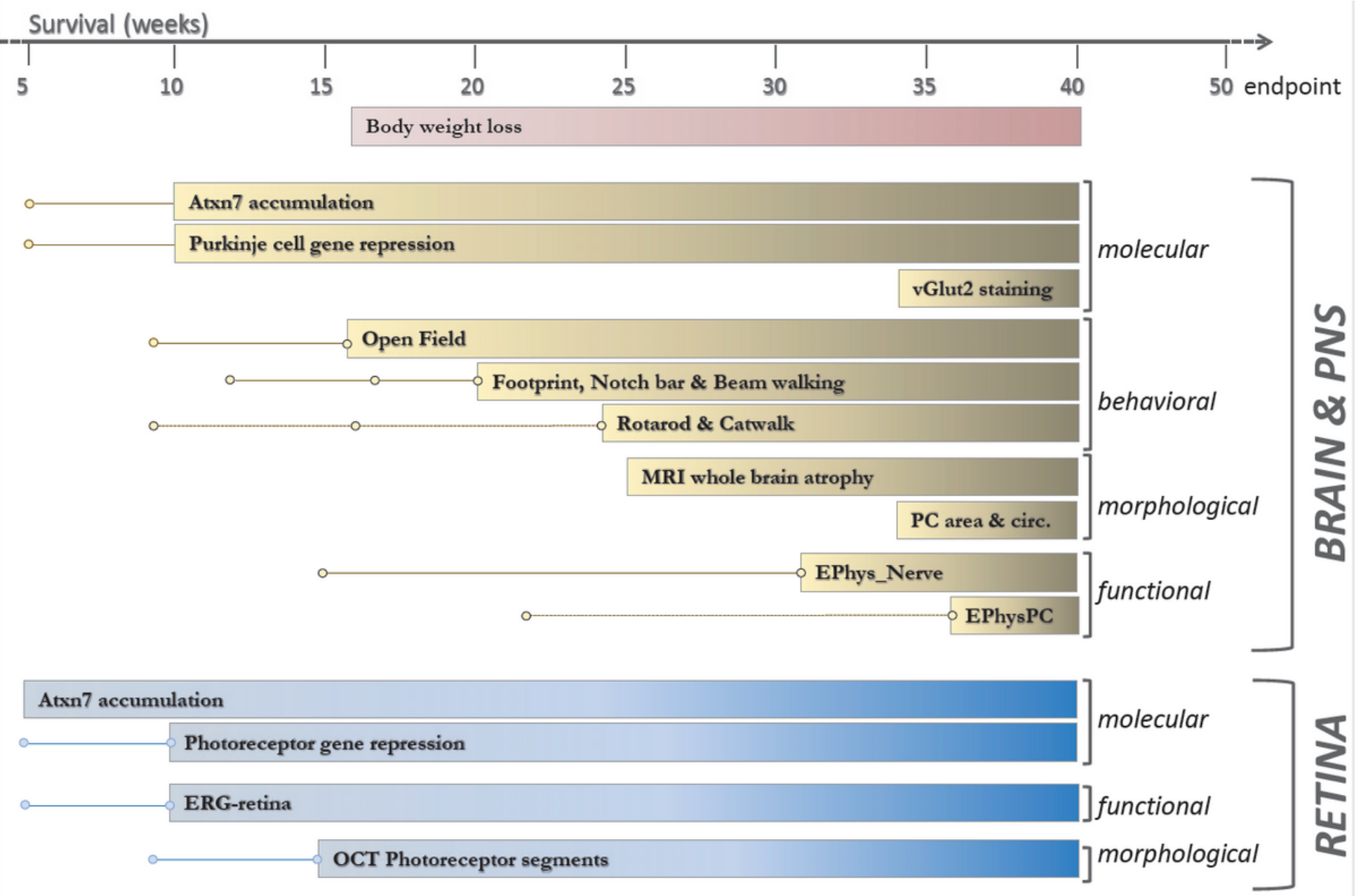

\section{Figure 10}

Summary of onset and progression of SCA7 mouse phenotypes. Dots and lines indicate analytic time points prior to phenotype onset. Bars correspond to phenotype onset and progression until 40 weeks of age.

\section{Supplementary Files}

This is a list of supplementary files associated with this preprint. Click to download.

- Additionalfile3Niewiadomskaetal.pdf

- Additionalfile9Niewiadomskaetal.xlsx

- Additionalfile10Niewiadomskaetal.xlsx

- Additionalfile7Niewiadomskaetal.xlsx

- Additionalfile8Niewiadomskaetal.xlsx

- Additionalfile4Niewiadomskaetal.xlsx

- Additionalfile5Niewiadomskaetal.xlsx

- Additionalfile6Niewiadomskaetal.xlsx 
- Additionalfile2Niewiadomskaetal.xlsx

- Additionalfile1Niewiadomskaetal.pdf 